\title{
Die natürlichen Grenzen der Ökonomie
}

\author{
Plädoyer für eine ökologische Schuldenbremse
}

\section{Einleitung}

Kann und soll die Wirtschaft immer weiter wachsen? Diese Frage, um die es seit den Diskussionen um die »Grenzen des Wachstums « in den 1970er Jahren für eine Weile sehr still geworden war, ist mit der Finanz- und Wirtschaftskrise wieder aufgetaucht. Schlagworte wie »Besser statt mehr ", »Wohlstand ohne Wachstum «, »Postwachstumsgesellschaft « und »Immer mehr ist nicht genug! « kursieren auf aktuellen Buchtiteln und Konferenzeinladungen. Auf den ersten Blick erscheint dies angesichts rasant gestiegener Staatsschulden und hoher Arbeitslosenraten in vielen Industrieländern absurd. Was, wenn nicht möglichst hohes Wachstum, soll diese Probleme überwinden? Entsprechend setzen politische Strategien auf nationaler und europäischer Ebene, etwa der Koalitionsvertrag der aktuellen Bundesregierung oder die neue europäische Wirtschaftsstrategie »Europe 2020 «, nach wie vor völlig selbstverständlich auf wirtschaftliches Wachstum als Voraussetzung für Wohlstand und die Lösung gesellschaftlicher Probleme.

Auf der anderen Seite mehren sich wachstumskritische Stimmen in Gesellschaft, Wissenschaft und Politik. ${ }^{1}$ Dass die Finanz- und Wirtschaftskrise selbst als Konsequenz einer Wirtschaftsweise und -politik interpretiert werden kann, in denen mit zweifelhaften Mitteln auf kurzfristigen maximalen Gewinn gesetzt wurde, gibt Anlass zu Verunsicherung. Die Zuspitzung globaler ökologischer Probleme und die erheblichen Schwierigkeiten der nationalen und internationalen Politik, sie zu lösen, aber auch die zunehmende soziale Spaltung vieler Gesellschaften verstärken diese Zweifel. Die Mehrheit der Bürger in Deutschland ist laut einer Emnid-Umfrage vom August 2010 nicht mehr davon überzeugt, dass Wirtschaftswachstum ihre Lebensqualität steigern wird, und wünscht sich eine »neue Wirtschaftsordnung «. ${ }^{2}$ Andererseits sind 74 Prozent der Deutschen der Meinung, dass ein hohes Wirtschaftswachstum notwendig ist, um Umwelt- und Klimaschutz finanzieren zu können. ${ }^{3}$

In Wissenschaft und Politik wächst das Interesse an einer ernsthaften Auseinandersetzung mit der Wachstumsfrage. Ausdruck dieser Entwicklung ist beispielsweise die Einrichtung der Enquete-Kommission » Wachstum, Wohlstand, Lebensqualität

1 Siehe z.B. Binswanger 2010; Booth 2004; Coyle 2011; Enderlein 2010; Fitoussi, Laurent 2008; Jackson 2009; Miegel 2010; Paech 2009 a; Schor 2010; Seidl, Zahrnt 2010 a; Victor 2008 .

2 ZEIT online vom 18. August 2010.

3 BMU, UBA 2010, S. 20.

Leviathan, 40. Jg., 2/2012, S. 11783 
- Wege zu nachhaltigem Wirtschaften und gesellschaftlichem Fortschritt in der Sozialen Marktwirtschaft « beim Deutschen Bundestag ${ }^{4}$ oder die Debatte um alternative Indikatoren zur Messung von Wohlstand, die vom früheren französischen Präsidenten durch die Einsetzung der Stiglitz-Sen-Fitoussi-Kommission angestoßen ${ }^{5}$ und von der deutschen Politik aufgegriffen wurde. ${ }^{6}$

Im folgenden Beitrag beschreiben wir das Wachstumsdilemma, dem sich heutige Gesellschaften in den reichen Ländern gegenübersehen. Wir setzen uns mit verschiedenen Lösungsansätzen in der aktuellen politischen Debatte auseinander und fragen nach deren theoretischer Fundierung in den Wirtschaftswissenschaften. Für einen konstruktiven Umgang mit dem Wachstumsdilemma empfehlen wir eine ökologische $»$ Schuldenbremse .

\section{Die Wachstumskontroverse}

\section{1 Ökonomische Gründe für Wachstum}

Aus ökonomischer Sicht spricht eine Reihe von Gründen dafür, wirtschaftliches Wachstum anzustreben und eine wachstumsorientierte Politik zu betreiben. Für Entwicklungs- und Schwellenländer wird die Notwendigkeit von wirtschaftlichem Wachstum bisher nicht in Frage gestellt, deswegen soll hier nur auf die ökonomischen Motive eingegangen werden, die für wirtschaftliches Wachstum in den früh industrialisierten Ländern sprechen. Fünf Gründe stehen dabei im Vordergrund. Die ersten beiden sind grundsätzlicher Natur, werden aber häufig übersehen; die letzten drei sind eng miteinander verbunden und dominieren in der Regel den Wachstumsdiskurs.

Einen wichtigen Grund für ökonomisches Wachstum hat Edmund S. Phelps in den Mittelpunkt seines Vortrags gestellt, als ihm 2006 der Preis für Wirtschaftswissenschaften der schwedischen Reichsbank im Gedenken an Alfred Nobel (» Wirtschaftsnobelpreis «) verliehen wurde. Von zentraler Bedeutung für eine »gute Wirtschaft « (»good economy «) sei es, dass sie möglichst vielen Menschen die Möglichkeit zu Partizipation und Selbstverwirklichung im Berufsleben gebe - durch die Teilhabe an Innovationsprozessen, durch das Bewältigen neuer Herausforderungen, durch die Stimulierung von Initiative, durch die Übernahme von Verantwortung et cetera. ${ }^{7}$ Eine Volkswirtschaft, welche diese Entfaltungsmöglichkeiten biete, benötige und generiere zugleich eine permanente Dynamik. Und diese Dynamik sei zu-

4 Siehe www.bundestag.de/bundestag/ausschuesse17/gremien/enquete/wachstum/index.jsp (Zugriff vom 30.04.2012).

5 Die international besetzte »Commission on the Measurement of Economic Performance and Social Progress « (Kommission zur Messung der wirtschaftlichen Leistungsfähigkeit und des sozialen Fortschritts) wurde Anfang 2008 von der französischen Regierung ins Leben gerufen und legte im September 2009 ihren Abschlussbericht vor (Stiglitz et al. 2009).

6 SVR, CAE 2010.

7 Phelps 2007, S. 556. 
mindest in der Vergangenheit untrennbar mit technischem Fortschritt und wirtschaftlichem Wachstum verbunden gewesen. Phelps und andere Ökonomen sind davon überzeugt, dass die von ihnen als "Degeneration « betrachtete Verwandlung von einer dynamischen Produktivgesellschaft zu einer statischen Rentiergesellschaft nur verhindert werden kann, wenn technischer Fortschritt und damit fast unvermeidlich einhergehendes Wachstum auch künftig möglich sind und angestrebt werden.

Eng benachbart zu Phelps' Argument ist die wirtschaftshistorisch begründete These von Benjamin M. Friedman, dass wirtschaftliches Wachstum nicht nur Verbesserungen der individuellen Lebenssituation ermöglicht, sondern auch eine positive gesellschaftliche Entwicklung befördert. Neben materiellen würden durch höheres Wachstum auch und gerade kulturelle Fortschritte auf gesellschaftlicher Ebene möglich, beispielsweise Toleranz, Fairness und eine Festigung der Demokratie. Eine unerlässliche Bedingung für das Eintreten dieser positiven Folgen sei jedoch, dass die große Mehrheit der Bevölkerung an den Früchten des Wachstums partizipiert. ${ }^{8}$ In einer Auseinandersetzung mit den USA und den drei großen europäischen Volkswirtschaften Großbritannien, Frankreich und Deutschland zeigt Friedman, dass zentrale gesellschaftliche Entwicklungsschritte und politische Richtungsentscheidungen - zum Guten wie zum Schlechteren - mit dem jeweiligen ökonomischen Kontext eng korrelieren: Gesellschaftlichen Fortschritten in Wachstumsphasen, in den USA etwa die Gesetzgebung zwischen 1895 und 1919 oder während der Bürgerrechtsbewegung zwischen 1945 und 1973, ${ }^{9}$ stehen dabei Rückschritte in Phasen der Stagnation gegenüber - mit dem auch durch die erste Weltwirtschaftskrise begünstigten Aufstieg der Nationalsozialisten in Deutschland zwischen 1930 und 1933 als markantestem historischen Beispiel. ${ }^{10}$

In der aktuellen Wachstumskontroverse stehen jedoch nicht diese beiden grundsätzlichen Gedankengänge, sondern vor allem drei konkrete Motive für Wachstum im Vordergrund. Danach ist Wirtschaftswachstum unabdingbar erstens für die Verfügbarkeit von Arbeitsplätzen, zweitens für die Finanzierung der sozialen Sicherungssysteme im Kontext des demografischen Wandels und drittens für die Rückführung der Staatsschulden auf ein tragfähiges Niveau.

Die Relevanz des Wirtschaftswachstums für die Verfügbarkeit von Arbeitsplätzen resultiert aus Fortschritten in der Arbeitsproduktivität. Bei konstanten Löhnen entstehen neue Arbeitsplätze nur dann, wenn die reale Wachstumsrate oberhalb der Zunahme der Arbeitsproduktivität liegt. Dieser Wert wird auch als »Beschäftigungsschwelle « bezeichnet. Weil die durch Rationalisierung erzielbaren Produktivitätsfortschritte im verarbeitenden Gewerbe größer ausfallen als im Dienstleistungssektor, ist die Beschäftigungsschwelle durch die zunehmende Bedeutung des

8 Vgl. Friedman 2005, S. 4.

9 Ebd., S. 214.

10 Ebd., S. 294; kritisch dazu unter anderem DeLong 2006. 
Dienstleistungsbereichs gefallen. ${ }^{11}$ In Ländern mit schrumpfender Bevölkerung wie Deutschland wird dieses Problem durch den demografischen Wandel tendenziell entschärft.

Für die anderen zwei Wachstumsmotive - Finanzierung der sozialen Sicherungssysteme im demografischen Wandel und Senkung der Schuldenlast - gilt, dass Wirtschaftswachstum diese eng miteinander verbundenen Aufgaben einfacher macht, für eine dauerhafte Lösung allein jedoch nicht ausreichend ist. So liegt der Schlüssel zur Bewältigung des demografischen Wandels vor allem darin, zusätzliche Lebensjahre so zwischen Erwerbs- und Rentenphase aufzuteilen, dass angemessene Rentenleistungen bei individuell akzeptablen und im internationalen Wettbewerb nicht zu hohen Beitragslasten erwirtschaftet werden können. ${ }^{12}$ Je geringer der Produktivitätsfortschritt ausfällt, umso größer wird tendenziell die Erhöhung des Renteneintrittsalters ausfallen müssen, um das Niveau der Rentenauszahlungen konstant halten zu können. Was für die Rente gilt, trifft näherungsweise auch auf die Krankenversicherung zu.

Von einer an den demografischen Wandel angepassten Ausrichtung sind die sozialen Sicherungssysteme in Deutschland derzeit noch weit entfernt: Die implizite Staatsschuld, welche die Leistungsversprechen des Staates ohne entsprechende Rücklagen oder Einnahmegrundlagen beziffert, beläuft sich nach aktuellen Simulationen in der Gesetzlichen Rentenversicherung auf 98,9 Prozent des BIP (Bruttoinlandsprodukt), in der Gesetzlichen Krankenversicherung auf 102,8 Prozent des BIP und in der Sozialen Pflegeversicherung auf 35,6 Prozent des BIP. ${ }^{13}$ Die jüngste Expertise des Sachverständigenrats zur Begutachtung der gesamtwirtschaftlichen Entwicklung (SVR) zu den Herausforderungen des demografischen Wandels kommt zu dem Ergebnis, dass Deutschland derzeit eine Tragfähigkeitslücke in Höhe von 3,1 Prozent des BIP aufweist. Um diesen Wert müssten die Primärsalden aus Staatseinnahmen und -ausgaben vor Zinszahlungen sofort und dauerhaft erhöht werden, um eine langfristige Tragfähigkeit der öffentlichen Haushalte sicherzustellen.

Bei der dritten Herausforderung, der Reduzierung der expliziten Schuldenlast, wird die vorhandene Wachstumsabhängigkeit dadurch augenfällig, dass zur Beurteilung der finanziellen Solidität eines Staates nicht der absolute akkumulierte Schuldenstand oder das absolute in einem Jahr ausgewiesene Budgetdefizit entscheidend sind, sondern jeweils deren Verhältnis zum BIP. Relational definierte Grenzwerte, wie im europäischen Stabilitäts- und Wachstumspakt drei Prozent für die Defizitquote und 60 Prozent für die Staatsschuldenquote, tragen auch der Tatsache Rechnung, dass die Wachstumsdynamik von großer Bedeutung für die langfristige Tragfähigkeit einer bestehenden Schuldenlast ist. Dies wird in der gegenwärtigen Ver-

11 Für Deutschland lag die Beschäftigungsschwelle, also die Wachstumsrate, die bei vereinfachter saldenmechanischer Betrachtung notwendig ist, um die Zunahme der Arbeitsproduktivität auszugleichen, empirischen Schätzungen zufolge in den 1960er Jahre zwischen 4,1 und 4,4 Prozent, in der Dekade von 1990 bis 2000 zwischen 1,1 und 2,4 Prozent (vgl. Schirwitz 2005, S. 36).

12 Vgl. Börsch-Supan 2011; SVR 2011.

13 Vgl. Raffelhüschen, Moog 2010, S. 12. 
schuldungskrise in der Eurozone besonders deutlich: Die unterschiedliche Bewertung von Staaten mit vergleichbarer Schuldenlast durch die Finanzmärkte ist unter anderem dadurch begründet, dass für die einzelnen Volkswirtschaften unterschiedliche Wachstumspotenziale diagnostiziert werden.

Es ist also kein Wunder, dass auch die Strategien zur Reduzierung übermäßiger Defizite und Staatsschulden meistens bei einer Steigerung des Wirtschaftswachstums ansetzen: Das Budgetdefizit ergibt sich ja als Differenz von Staatsausgaben und Staatseinahmen. Um die Defizitquote zu verringern, muss entweder das Budgetdefizit verkleinert oder das BIP gesteigert werden. Versucht die Politik ersteres, hat sie die Wahl zwischen Leistungskürzungen, Steuer- und Abgabenerhöhungen oder einer Kombination dieser gleichermaßen unpopulären Maßnahmen. Eine wachstumsförderliche Wirtschaftspolitik ist vor diesem Hintergrund eine attraktive und Verteilungskonflikte vermeidende Alternative. ${ }^{14}$

Die jüngere Wirtschaftsgeschichte in Deutschland und in vielen anderen OECDStaaten zeigt allerdings, dass wirtschaftliches Wachstum allein keinesfalls solide Staatsfinanzen garantiert. Denn die Verschuldungsbereitschaft der Politik war stets systematisch größer als die erzielten Wachstumsraten. Verantwortlich dafür war nicht ausbleibendes Wachstum, sondern entscheidend waren institutionelle Defizite und ein Mangel an langfristig orientierter Verantwortung in der Politik. Die im Jahr 2009 im Grundgesetz verankerte sogenannte Schuldenbremse trägt dieser Tatsache dadurch Rechnung, dass die von Konjunkturschwankungen unabhängige strukturelle Verschuldung ${ }^{15}$ des Bundes ab 2016 auf 0,35 Prozent des BIP beschränkt ist und die Bundesländer ab 2020 gar keine strukturellen Schulden mehr machen dürfen. Die bisher oft genutzte Hintertür, Staatsausgaben als Investitionen zu deklarieren, die keine echten Investitionen waren, und darüber neue Schulden zu ermöglichen, soll die Schuldenbremse versperren. Damit die Schuldenbremse nicht zur Investitionsbremse wird, muss die Politik die Mittel für Investitionen künftig entweder durch die Kürzung anderer Ausgaben oder durch Steuererhöhungen finanzieren, wenn sie nicht auf höhere Einnahmen infolge einer wachsenden Wirtschaft zurückgreifen kann. Es spricht folglich viel dafür, dass die Wachstumsorientierung zumindest der deutschen Politik eher noch zunimmt, sobald die Konsequenzen der Schuldenbremse in ihrer ganzen Tragweite erkannt und berücksichtigt werden. ${ }^{16}$

14 Gebhardt et al. 2009. Allerdings stellt der Sachverständigenrat bezüglich der Sanierung der Staatsfinanzen zutreffend fest: "Ein höheres Wachstum erleichtert die Konsolidierungsaufgabe also, kann sie aber keinesfalls lösen. Umgekehrt würde ein geringeres dauerhaftes Wachstum alles noch schwieriger machen«(SVR 2009, S. 186).

15 Das strukturelle Defizit bezeichnet den Teil des Defizits der öffentlichen Haushalte, der dauerhaften Charakter hat, sich also nicht im Lauf eines Konjunkturzyklus ausgleicht oder durch gesetzlich befristete Maßnahmen begründet ist, und der den mittelfristig als unbedenklich erscheinenden Umfang staatlicher Kreditfinanzierung überschreitet (SVR 1994, S. 152).

16 Vgl. RWI 2010; SVR 2009, S. 179 ff.

Leviathan, 40. Jg., 2/2012 


\subsection{Grenzen des Wachstums aus ökologischer Sicht}

Den guten Gründen für das Wachstum aus ökonomischer Perspektive steht eine ebenso gut begründete Skepsis gegenüber, die sich wesentlich aus der Sorge um die Ökologie des Planeten speist. Die Umweltwissenschaft liefert vermehrt Hinweise dafür, dass die globale Wirtschaft eine Größenordnung erreicht, die die Belastbarkeit der natürlichen Systeme sprengt. Der Klimawandel schreitet voran, die Meeresspiegel steigen, Gletscher schmelzen und extreme Wetterereignisse häufen sich. Der Verlust biologischer Vielfalt konnte bisher nicht einmal verlangsamt, geschweige denn gestoppt werden. Etwa 60 Prozent der Ökosysteme sind bereits degradiert oder durch nicht-nachhaltige Nutzung gefährdet. Wertvolle Ökosysteme wie Tropen- und Mangrovenwälder oder Korallenriffe gehen verloren. Unter den Folgen nicht-nachhaltiger Wasser- und Bodennutzung leiden bereits heute viele Regionen der Erde, in Zukunft werden immer mehr Menschen davon betroffen sein. ${ }^{17}$

Das Verständnis der ökologischen Grenzen des Wachstums hat sich seit den 1970er Jahren stark entwickelt. Damals drehte sich die Diskussion in erster Linie um die Frage, ob und wann die Begrenztheit natürlicher Rohstoffvorkommen dem Wachstum ein Ende setzen würde. Die Autoren des Berichts über die »Grenzen des Wachstums « 18 wurden insbesondere dafür kritisiert, die Anpassungsfähigkeit der Märkte nicht angemessen berücksichtigt und deshalb die Auswirkungen von Rohstoffverknappung auf die wirtschaftliche Gesamtentwicklung überschätzt zu haben. ${ }^{19}$ Nach wie vor sind die Einschätzungen zur Rohstoffverknappung kontrovers. ${ }^{20}$

Dagegen gibt es heute einen weitgehenden Konsens in den Umweltwissenschaften, dass ökologische Grenzen für die materielle Ausdehnung der Ökonomie existieren und dass diese eher in der Gefährdung der Funktionsfähigkeit natürlicher Systeme (z.B. stabiles Klima, Ökosysteme, Wasserkreislauf) liegen als in der Verfügbarkeit einzelner nichterneuerbarer Rohstoffe. Die Bedeutung erneuerbarer Ressourcen als Produktionsgrundlage, aber auch als »ökologischer Dienstleister « (z.B. für die Aufnahme von Treibhausgasen) und ihre Regenerationsfähigkeit stehen im Vordergrund.

Neuere Ansätze, die die Grenzen des Wachstums zu bestimmen versuchen, widmen sich verstärkt dem Umgang mit Unsicherheit, mit nichtlinearen Prozessen und der Problematik normativer Bewertung. Wie sicher können die Reaktionen natürlicher Systeme auf Belastungen bestimmt werden? Wie viel Risiko kann eine Gesellschaft bzw. ihre politische Führung übernehmen? Die Grenzen der Umweltbelastung können angesichts der Komplexität der Systeme und Prozesse grundsätzlich

17 CBD 2010; IPCC 2007 a; Millenium Ecosystem Assessment 2005; UNEP 2007; WWF et al. 2010.

18 Meadows et al. 1972.

19 Siehe z.B. Cole et al. 1973.

20 Vgl. z.B. BGR 2009; Frondel, Schmidt 2007; Kavalov, Peteves 2007; UNEP, IPSRM 2010; Zittel, Schindler 2007. 
nicht als klare Linie gedacht, sondern müssen als »Korridore der Elastizität « 21 gesehen werden. Werden bestimmte »Umkipp-Punkte « überschritten, können abrupte und irreversible Veränderungen eintreten und Systeme von einem stabilen Zustand in einen anderen übergehen. ${ }^{22}$ Das klassische Beispiel aus der Ökologie ist der See, der » umkippt «, wenn die Nährstoffbelastung einen bestimmten Wert überschreitet, und dadurch die vorher charakteristische und stabile Artenzusammensetzung verliert. Auch in der Klimawissenschaft spielen »Umkipp-Punkte « eine wichtige Rolle. So könnte etwa das Inlandeis Grönlands vollständig abschmelzen, wenn eine bestimmte Stufe der Erwärmung erreicht wird, was zu einem erheblich stärkeren Meeresspiegelanstieg und einer Abschwächung des Golfstroms führen würde. ${ }^{23}$ Die Grenzen des Wachstums sind nach diesem Verständnis dann erreicht, wenn die Widerstandsfähigkeit (»Resilienz «) natürlicher Systeme beeinträchtigt wird - also ihre Fähigkeit, sich von Störungen und Schocks zu erholen und dabei ihre Funktionen aufrechtzuerhalten. ${ }^{24}$

Werden ökologische Grenzen und »Umkipp-Punkte « überschritten, gefährdet dies letztendlich auch die Wirtschaft der Menschen, die auf die Natur angewiesen ist. Zu den »Dienstleistungen der Natur " gehören Nahrung und Wasser, Biomasse für unterschiedlichste Zwecke, die Klimaregulierung, die Aufrechterhaltung von Nährstoffkreisläufen oder die Bestäubung von Nutzpflanzen durch Insekten. ${ }^{25}$ Die Menschheit nutzt diese »Dienstleistungen der Natur « heute intensiver als je zuvor und ist dadurch in der Lage, einen höheren materiellen Lebensstandard für viele Menschen zu erreichen und eine nie gekannte wirtschaftliche Leistung zu erbringen. Gleichzeitig sind mit diesem Wirtschaftsprozess aber auch eine wachsende Degradierung von Ökosystemen und der häufig irreversible Verlust der »Dienstleistungen der Natur « verbunden. ${ }^{26}$ Die Erosion der physischen Grundlagen wirtschaftlicher Prozesse wird aller Voraussicht nach ab einem bestimmten Punkt auch deren Wachstum beeinträchtigen und kann zu katastrophalen Zusammenbrüchen ökonomischer Systeme führen. ${ }^{27}$

Menschliche Wirtschaftsaktivitäten überschreiten bereits heute ökologische Belastbarkeitsgrenzen. Darauf weisen verschiedene Indikatorensysteme hin. So zeigt etwa die Entwicklung des »ökologischen Fußabdrucks", dass die Menschheit inzwischen mehr natürliche Ressourcen in Anspruch nimmt, als die Erde auf Dauer bereitstellen kann - Tendenz steigend. ${ }^{28}$ Rockström et al. haben versucht, die ökologischen Belastungsgrenzen (planetary boundaries) für einzelne natürliche Systeme und Prozesse zu quantifizieren. Sie schlagen sichere Bereiche für menschliche Akti-

21 Sachs, Santarius 2005, S. 33.

22 Lenton et al. 2008.

23 IPCC $2007 \mathrm{~b}$.

24 Walker, Salt 2006.

25 Millenium Ecosystem Assessment 2005.

26 Stern et al. 2007; TEEB 2010.

27 Meadows et al. 2006.

28 WWF et al. 2010; Wackernagel, Rees 1996. 
vität (einen safe operating space) vor, die jeweils ausreichend weit von möglichen »Umkipp-Punkten« oder gefährlichen Belastungsniveaus entfernt sind. Beim Klimawandel, dem Verlust von biologischer Vielfalt und in Bezug auf Eingriffe in den globalen Stickstoffkreislauf seien diese Sicherheitsleitplanken heute bereits deutlich überschritten, andere Belastungen etwa beim Phosphorkreislauf, bei der Versauerung der Ozeane, Landnutzung und Süßwassernutzung näherten sich den Grenzen an. ${ }^{29}$

Die zunehmende Umweltbelastung wird durch Bevölkerungswachstum und das Wachstum der Wirtschaft angetrieben - das zeigt ein Blick auf historische Zeitreihen und Ländervergleiche. Energieverbrauch, Treibhausgasemissionen, Ressourcenverbrauch und ökologischer Fußabdruck sind weltweit seit Beginn der Industrialisierung parallel zum Wachstum von Bevölkerung und Wirtschaftsleistung angestiegen. Der globale Trend ist nach wie vor ungebrochen, trotz gewisser Erfolge bei der Reduzierung des Rohstoffverbrauchs und der Kohlenstoffemissionen in einzelnen Ländern wie Deutschland. Die Gesamtbelastung nimmt also zu, auch wenn Ressourcen immer effizienter genutzt werden - Effizienzsteigerungen wurden vom Wachstum systematisch überholt. Zu diesem Phänomen trägt das sogenannte Jevons-Paradox bei, auch als Rebound-Effekt bekannt: Führen neue Technologien zu höherer Effizienz einer Ressourcennutzung, dann reduzieren sie deren Preis, was wiederum zu einer höheren Nachfrage führt. Dass der Rebound-Effekt regelmäßig relevante Größenordnungen erreicht und Effizienzgewinne zu erheblichen Anteilen ausgleicht, ist umfassend nachgewiesen. ${ }^{30}$

Angesichts dieser zunehmenden Bedrohlichkeit weltweiter Umweltbelastung, die ganz offensichtlich eng mit dem Bevölkerungswachstum und dem steigenden materiellen Lebensstandard zusammenhängt, stellt sich die zentrale Frage, ob weiteres Wachstum mit den ökologischen Begrenzungen in Einklang gebracht werden kann.

\subsection{Wirtschaften ohne Wachstum? Vorschläge in der aktuellen Debatte}

Tim Jackson prägte für die Situation, in der sich reiche Länder heute befinden, den Begriff »Wachstumsdilemma «: ${ }^{31}$ Einerseits scheinen Volkswirtschaften auf Wachstum angewiesen zu sein. Es ist derzeit schwer vorstellbar, wie in der heutigen globalisierten Wirtschaft Stagnation oder Schrumpfen stabil sein könnten, da sinkende Nachfrage, steigende Arbeitslosigkeit, zunehmende Staatsverschuldung und abnehmende Wettbewerbsfähigkeit sich gegenseitig verstärken und in eine Abwärtsspirale führen würden. Andererseits bedeutet fortgesetztes Wachstum eine steigende Umweltbelastung und birgt so das Risiko, natürliche Lebensgrundlagen unwiederbringlich zu zerstören. Um Auswege aus diesem Wachstumsdilemma kreist die aktuelle Debatte. Dabei bewegen sich die Lösungsansätze zwischen zwei Polen: »Green Growth «, einem ökologisch verträglichen Wachstum auf der einen Seite, und »Post-

29 Rockström et al. 2009.

30 Siehe z.B. Schor 2010; Barker et al. 2009; Holm, Englund 2009; Frondel et al. 2008; Sorrell 2007.

31 Jackson 2009. 
wachstum « oder » Degrowth « auf der anderen Seite - Positionen, die das Wachstum selbst oder zumindest seine Vorherrschaft als politisches Ziel in Frage stellen.

\section{Wie weit trägt Green Growth? Perspektiven und Grenzen der Entkopplung}

»Green Growth « oder das Wachstum einer »Green Economy « stehen derzeit hoch im Kurs. ${ }^{32}$ Das Wachstum der Wirtschaft soll vom Umweltverbrauch »entkoppelt " werden, indem Energie und Material effizienter genutzt und neue Technologien mit geringerer Umweltauswirkung eingesetzt werden oder auch indem Konsummuster sich von ressourcenintensiven Produkten zu »immateriellen« Dienstleistungen hin verschieben.

Die Spielräume für die Entkopplung von Wirtschaftsleistung und Naturverbrauch durch technologischen Fortschritt sind mit Sicherheit groß und bislang nicht im Entferntesten ausgeschöpft. Steigerungen von Energie- bzw. Ökoeffizienz um den Faktor vier, fünf oder zehn sind in vielen Produktionsprozessen möglich. ${ }^{33}$ Szenariostudien weisen darauf hin, dass weltweit eine Energieversorgung weitgehend ohne Treibhausgasemissionen möglich wäre - und dass die dafür notwendige technologische Transformation nicht zu nennenswerten Einbußen beim Wachstum führen müsste. ${ }^{34}$ Wichtige Bereiche der Energieversorgung könnten prinzipiell vollständig oder weitgehend auf erneuerbare Energieträger umgestellt werden - in Deutschland, Europa und sogar weltweit. ${ }^{35}$ Die Energieversorgung könnte demnach mit vorhandenen Technologien von den Treibhausgasemissionen entkoppelt werden - falls dies gesellschaftlich gewünscht und politisch durchgesetzt würde.

Dennoch sehen sich auch Green-Growth-Strategien großen Herausforderungen gegenüber. Vereinfacht betrachtet führt Green Growth nur dann zu tatsächlich sinkender Umweltbelastung, wenn die Entkopplungsrate dauerhaft größer ist als die Wachstumsrate der Wirtschaft. ${ }^{36}$ Andernfalls werden die Verbesserungen bei der Ökoeffizienz durch das Wachstum und Rebound-Effekte überholt. Insofern ist auch bei Green Growth die Wachstumsrate der Wirtschaft (bzw. ihr Verhältnis zur Entkopplungsrate) eine relevante Größe - eine unbequeme Einsicht, die häufig unter den Tisch fällt. Zudem ist die Entkopplungsdiskussion häufig auf einzelne Probleme (etwa den Klimaschutz) konzentriert und kümmert sich nicht immer ausreichend um mögliche Problemverlagerungen. So bringt etwa eine emissionsarme Energieversorgung auf der Basis von Atomenergie oder Kohlendioxydspeicherung (carbon capture and storage, CCS) neue Umweltprobleme und -risiken mit sich. Die Nut-

32 OECD 2010; UNEP 2010.

33 Schmidt-Bleek 1997; von Weizsäcker et al. 2010.

34 Vgl. z.B. Edenhofer et al. 2010.

35 Öko-Institut, Prognos AG 2009; SRU 2011; EREC 2010; ECF et al. 2010; PwC et al. 2010; FoEE, SEI 2009; EREC, Greenpeace International 2010; WWF et al. 2011.

36 Victor 2010; Huesemann 2003, S. 30.

Leviathan, 40. Jg., 2/2012 
zung erneuerbarer Energien erhebt Anspruch auf die ebenfalls knappe Ressource Fläche und kann so mit negativen ökologischen Auswirkungen verbunden sein. ${ }^{37}$

Es ist also eine offene Frage, wie weit Entkopplung tragen kann und wo ihre thermodynamischen bzw. physikalischen Grenzen liegen. Die Abhängigkeit wirtschaftlichen Wachstums von einer steigenden Zufuhr an hochwertiger Energie ist wissenschaftlich gut belegt; ${ }^{38}$ Wirtschaften ist nichts anderes als ein permanenter Stoffwechsel mit der Natur. ${ }^{39}$ Die Voraussetzung für dauerhaft nachhaltiges Wachstum wäre dementsprechend ein rascher und vollständiger Umstieg auf erneuerbare Energiequellen, die so effizient und ökologisch optimiert wie möglich genutzt werden müssten. Der Verbrauch materieller natürlicher Ressourcen müsste ebenfalls durch die Nutzung dieser erneuerbaren Energie, beispielsweise für Gewinnung und Recycling von Rohstoffen oder die Produktion erneuerbarer Ressourcen, reduziert werden. Was dies angesichts des hohen Flächenbedarfs erneuerbarer Energien und der Konkurrenz mit anderen Flächennutzungen für die Wirtschaft und ihr Wachstum bedeuten würde, ist nicht eindeutig geklärt.

Die bisherige enge Kopplung von Wirtschaftsleistung und Umweltbelastung (siehe Abschnitt 2.2) illustriert, welche Herausforderung ein »echtes « Green Growth darstellt. Annahmen, denen zufolge ökologische Probleme sich bei steigenden Einkommen von selbst lösen (Umwelt-Kuznets-Kurve) oder denen zufolge in den reichen Ländern das Wachstum bereits heute vorwiegend qualitativer Natur sei, ${ }^{40}$ sind angesichts der empirischen Daten nicht haltbar: Bisher wachsen absoluter Energie- und Ressourcenverbrauch weiter. Die Zuwächse nehmen bestenfalls leicht ab oder der Verbrauch stagniert in Ländern mit einer besonders engagierten Umweltpolitik wie Deutschland, ohne dass das auch hierzulande nach wie vor viel zu hohe Niveau wirklich sinkt. Zudem ergibt sich häufig ein anderes Bild, wenn Handelsbilanzen und damit Warenimporte berücksichtigt werden, für deren Herstellung die Natur in den Herkunftsländern geschädigt wurde, also die sogenannten importierten »Rohstoffrucksäcke «. ${ }^{41}$ Kuznets-Effekte lassen sich für lokale Wasser- und Luftverschmutzung beobachten, nicht jedoch für globale und weniger unmittelbar sichtoder spürbare Belastungen wie Klimawandel und Biodiversitätsverlust. ${ }^{42}$ Auch gibt es bisher keine Hinweise darauf, dass höhere Wachstumsraten den Umweltschutz durch schnellere Entkopplung vorantreiben. ${ }^{43}$

Green Growth erscheint als Lösungsvorschlag konzeptionell attraktiv, insbesondere da es problemlos mit dem bisherigen Welt- und Wirtschaftsbild vereinbar ist. Es erfordert zwar erhebliche Veränderungen im Bereich der Technologie, aber keine grundsätzlicheren Änderungen bei Kultur, Lebensstilen, Konsum- und Wirtschafts-

37 SRU 2011, Tz. $53 \mathrm{ff}$.

38 Siehe z.B. Boulding 1966; Frondel, Schmidt 2004; Ayres, Warr 2010; Sorrell 2010.

39 Lowe 1965, S. 20.

40 Paqué 2010.

41 Helm et al. 2007; Statistisches Bundesamt 2010.

42 Vgl. z.B. Shafik, Bandyopadhyay 1992; Victor 2010, S. 241.

43 Victor 2010. 
gewohnheiten. Allerdings bleibt Green Growth im Sinne einer umfassend von den Umweltauswirkungen entkoppelten Gesamtwirtschaft bislang eine Wunschvorstellung, von der die Realität weit entfernt ist.

\section{Postwachstumsgesellschaften: Lösungsansätze fragmentarisch}

Die eher wachstumskritischen Autoren halten Green Growth für nicht ausreichend. Sie eint die Befürchtung, dass eine hinreichende Entkopplung des Wirtschaftswachstums von seiner materiellen und energetischen Basis nicht gelingen wird. Während einige explizit ein Schrumpfen der Wirtschaft als Voraussetzung für nachhaltiges Wirtschaften fordern (Degrowth - Decroissance) ${ }^{44}$ steht bei den meisten die Forderung nach mehr Unabhängigkeit vom Wachstum im Vordergrund. Gesellschaften sollten sich auf geringere Wachstumsraten einstellen bzw. in der Lage sein, auch ohne Wachstum zu funktionieren. ${ }^{45}$ Dies nicht nur, weil ökologische Grenzen dies notwendig machen, auch die Tendenz sinkender Wachstumsraten in Deutschland und anderen reichen Industrieländern, ${ }^{46}$ Zweifel an der Möglichkeit unendlichen exponentiellen Wachstums ${ }^{47}$ oder die abnehmende Bedeutung von Einkommenssteigerungen in reichen Gesellschaften für Lebensqualität und Zufriedenheit ${ }^{48}$ sind zentrale Argumente. Neue, stärker qualitativ ausgerichtete Paradigmen, etwa das Ziel einer nachhaltigen Entwicklung, sollen das Wachstum des BIP als politische Priorität ablösen.

Die Herausforderung an eine Theorie der Postwachstumsgesellschaft oder Postwachstumsökonomie besteht darin, Lösungen für das Wachstumsdilemma zu finden. Wie können Staaten und Gesellschaften mit den Risiken ausbleibenden Wachstums oder womöglich einer schrumpfenden Ökonomie umgehen? Wie können gesellschaftliche Institutionen und Systeme vom Wachstum unabhängiger gemacht werden? Die Lösungsansätze der Postwachstumsliteratur hierzu sind allerdings bislang höchst fragmentarisch und nicht weit genug gediehen, um als echte Alternative für politisches Handeln dienen zu können. Dies kann an zwei Problemfeldern deutlich gemacht werden (siehe Abschnitt 2.1): der Beschäftigungssicherung und der Staatsfinanzierung.

Der in der Regel positive Zusammenhang von Wirtschaftswachstum und Beschäftigung ist eines der stärksten Argumente für Wachstum, da die Beschäftigungssituation sowohl für die Lebenszufriedenheit des Einzelnen als auch für die soziale Stabilität von Gesellschaften eine zentrale Rolle spielt. Beschränkungen des Wirtschaftswachstums würden bei weiterer Zunahme der Arbeitsproduktivität zu höherer Arbeitslosigkeit führen. In der Postwachstumsliteratur findet sich als Antwort

44 Siehe z.B. Flipo, Schneider 2008.

45 Jackson 2009; Miegel 2010; Seidl, Zahrnt 2010 a; Sachs 2010.

46 Reuter 2010.

47 Enderlein 2010; Diefenbacher, Zieschank 2009, S. 21.

48 Jackson 2009.

Leviathan, 40. Jg., 2/2012 
auf dieses Problem häufig die Empfehlung, die Arbeitszeit zu verkürzen - zum Beispiel durch verringerte Wochenstunden oder weniger Arbeitstage - und die vorhandene Arbeit gerechter zu verteilen. ${ }^{49}$

Auf den ersten Blick erscheint es vielleicht plausibel, Fortschritte bei der Arbeitsstundenproduktivität durch eine Reduzierung der Jahresarbeitszeit so zu neutralisieren, dass auch bei konstantem BIP keine Reduzierung der Erwerbstätigenzahl resultiert. Dieser Strategie liegt jedoch die falsche Annahme zu Grunde, dass die Arbeitsmenge in einer Volkswirtschaft konstant ist. Die Identität, dass die Veränderungsrate des BIP gleich der Summe der Veränderungsraten von Arbeitsstundenproduktivität, Jahresarbeitszeit und Erwerbstätigenzahl ist, gilt erst ex post - tatsächlich ist die Arbeitsnachfrage in einer Volkswirtschaft nicht gegeben, sondern entsteht selbst erst im ökonomischen Prozess unter Einfluss verschiedenster Faktoren. Das Aufteilen eines als konstant angenommenen Arbeitsvolumens kann deshalb dazu führen, dass nicht die Arbeitslosigkeit, sondern das BIP sinkt, wie empirische Ergebnisse bestätigen. ${ }^{50}$ Darüber hinaus dürfte eine solche Strategie in Konflikt mit der Bewältigung des demografischen Wandels geraten, welche mit Fortschreiten der Lebenserwartung auch längere Lebensarbeitszeiten erfordert. ${ }^{51}$

Auf der anderen Seite war über lange Zeiträume in der Vergangenheit eine Abnahme der jährlichen Arbeitszeit pro Person zu beobachten. In der konkreten Aufteilung der Produktivitätsgewinne auf mehr Freizeit einerseits und höhere Löhne andererseits zeigen sich im internationalen Vergleich jedoch deutliche Unterschiede, beispielsweise zwischen Europa und den USA. ${ }^{52}$ Solange die Summe aus Lohn- und Freizeitzuwachs nicht über den Zuwachs an Produktivität hinausgeht, bestehen hier auch im Kontext der Globalisierung weiterhin Spielräume. Vor diesem Hintergrund erscheint es sinnvoll, flexible Arbeitszeitmodelle zu fördern und es Arbeitnehmern zu erleichtern, sich freiwillig für kürzere Arbeitszeiten (pro Woche, Monat oder Jahr) in einer insgesamt längeren Lebenserwerbsphase zu entscheiden. Wenn es in einem flexiblen Regime gelänge, für die bisher Beschäftigten mehr Freiheitsgrade zu verwirklichen und gleichzeitig die Erwerbsbeteiligung insbesondere von Frauen zu erhöhen, dann könnten neue Spielräume entstehen, um gesamtwirtschaftliche Erfordernisse sowie individuelle Bedürfnisse und ökologische Restriktionen besser miteinander in Einklang zu bringen. Statt auf die Reduzierung des ökologischen Fußabdrucks infolge einer quasi erzwungenen Verkürzung der Arbeitszeit und damit einhergehender Einkommensverluste zu setzen, ${ }^{53}$ würden die individuellen Möglichkeiten für eine nachhaltigere Lebensweise verbessert. ${ }^{54}$

Ein weiterer wichtiger Wachstumszwang moderner Gesellschaften liegt in der Dynamik der Staatsverschuldung (vgl. Abschnitt 2.1). Öffentliche Haushalte wer-

49 Vgl. z.B. Jackson 2009; Scherhorn 2010; Schor 2010.

50 Vgl. MEA 2007.

51 SVR 2011.

52 Schor 2010.

53 Nässén et al. 2009.

54 »Work less, spend less, emit and degrade less«; Schor 2010, S. 112. 
den in der Regel in der Erwartung wachsender Einnahmen geplant, so dass die Solidität der Finanzen später auf das Eintreten genau dieser Erwartungen angewiesen ist. Vorsätze, in Zeiten guter Konjunktur die Schulden abzuzahlen, fallen systematisch der politischen und gesellschaftlichen Versuchung zum Opfer, stattdessen die Ausgaben weiter zu erhöhen. Die ausufernde Staatsverschuldung ist eine der großen Herausforderungen, und sie ist schwer zu bewältigen, selbst wenn an Wachstumserwartungen festgehalten wird. Entsprechend erstaunt es nicht, dass auch die Wachstumsskeptiker hierzu keine Lösungen parat haben. Die Herausgeberinnen der 2010 erschienenen »Postwachstumsgesellschaft « konnten keinen Experten für einen Beitrag zum Thema Staatsfinanzen in der Postwachstumsgesellschaft finden. ${ }^{55} \mathrm{Ihr}$ eigener Text beschränkt sich auf einen Überblick zur aktuellen Diskussion und deutet Handlungsempfehlungen nur an - etwa Selbstbindungen der Politik durch Mechanismen wie die Schuldenbremse.

\subsection{Zwischenfazit}

Das Wachstumsdilemma prägt die Diskussion um die Grenzen des Wachstums seit ihren Anfängen. ${ }^{56}$ Inzwischen gewinnt es durch die Verschärfung der Umweltprobleme im globalen Maßstab und durch die zunehmende Komplexität der Zusammenhänge in einer globalisierten Wirtschaft an Brisanz. Aus ökologischer Perspektive hat der Metabolismus mit der Natur seinen Gleichgewichtspunkt bereits überschritten. Weiteres Wachstum unter Beibehaltung der bestehenden Produktionsund Konsummuster wird unweigerlich weiter an der Substanz der Natur zehren und die Resilienz der natürlichen Systeme weiter schwächen. Andererseits ist das bestehende Wirtschaftssystem nicht »resilient " gegenüber ausbleibendem Wachstum: Wesentliche gesellschaftliche Ziele scheinen ohne Wachstum nicht erreichbar zu sein.

Weder die Vorstellung vom Green Growth noch die Ansätze einer Postwachstumsgesellschaft geben eine befriedigende Antwort auf das Wachstumsdilemma. Die Postwachstumsliteratur weist auf ungelöste Konflikte zwischen Ökonomie und Ökologie hin und bietet interessante Ansatzpunkte und Ideen, die jedoch in der Konsequenz schwierige Fragen aufwerfen. Sie liefert bislang keine in sich schlüssigen Konzepte. Eine integrierte Perspektive auf Maßnahmen für Wachstumsunabhängigkeit, ihre Folgen und Wechselwirkungen miteinander fehlt, weshalb die Konzepte eher vage bleiben und für die Politik bisher kaum anwendbar sind.

Im folgenden Abschnitt wird gefragt, inwieweit die Wirtschaftswissenschaft eine angemessene theoretische Fundierung für die Wachstumsdebatte bereitstellt und ob sie die fehlende Konzeptionalisierung liefern kann.

55 Seidl, Zahrnt 2010 b.

56 Steurer 2004.

Leviathan, 40. Jg., 2/2012 


\section{3. Ökologische Grenzen in der ökonomischen Theorie}

\subsection{Neoklassische Ökonomie und Umwelt-und Ressourcenökonomie}

Beim Rückblick auf die Entwicklung der Wirtschaftswissenschaften fällt auf, dass biophysische Aspekte in der klassischen Nationalökonomie noch eine wichtige Rolle spielten. So sahen Thomas Malthus und David Ricardo in der begrenzten Verfügbarkeit fruchtbaren Bodens bzw. der abnehmenden Produktivität neu hinzukommender Böden eine Begrenzung für das Bevölkerungswachstum. ${ }^{57}$ In der klassischen ökonomischen Theorie war auch die Vorstellung verbreitet, dass die Wirtschaft aufgrund natürlicher Grenzen irgendwann einen stationären Zustand erreichen müsse. Der britische Philosoph und Ökonom John Stuart Mill hielt diesen stationären Zustand für unausweichlich, sah darin aber keinesfalls das Ende des Projekts, das menschliche Leben zu verbessern, sondern seine Verlagerung - weg von einer Steigerung der materiellen Produktion hin zu immateriellen Werten wie Kultur, Moral und sozialem Fortschritt. ${ }^{58}$ Auch John Maynard Keynes war von dieser Entwicklung hin zu einer gesättigten stationären Wirtschaft überzeugt, hat sie allerdings als Vision beschrieben. ${ }^{59}$ Die Wachstumstheorien der Klassiker wurden jedoch angesichts der Tatsache, dass weder das Bevölkerungswachstum noch das Wirtschaftswachstum ihren Prognosen gemäß abnahm, verworfen, und mit ihnen verschwand die Vorstellung von natürlichen Grenzen jeglicher Art weitgehend aus der ökonomischen Theorie.

Der heute relevante Kanon an wirtschaftswissenschaftlichen Wachstumsmodellen lässt sich in vier aufeinander aufbauende Paradigmen gliedern: ${ }^{60}$ Das neoklassische Wachstumsmodell wurde zu einem endogenen Wachstumsmodell, dem AK-Modell, erweitert; das erstmals Innovationen in den Mittelpunkt rückende ProductVariety Model bildete wiederum die Grundlage für die Entwicklung des Schumpeter'schen Wachstumsmodells. Natürliche Ressourcen spielen in den Basis-Varianten dieser vier Modelle jedoch keine eigenständige Rolle mehr.

Im neoklassischen Wachstumsmodell hängt die Produktion nur von zwei Faktoren ab: menschlicher Arbeit und Kapital. In der Regel wird eine hohe wechselseitige Ersetzbarkeit (Substitutionselastizität) zwischen diesen Produktionsfaktoren angenommen. Kapital steht dabei für von Menschen hergestellte Produktionsmittel, die ihrerseits zur Produktion von Gebrauchsgütern verwendet werden. Das Modell geht von einem abnehmenden Grenzertrag aus: Mit wachsendem Einsatz eines Produktionsfaktors nimmt bei konstantem Einsatz aller anderen Faktoren der Ertrag der nächsthöheren Einheit immer weiter ab. ${ }^{61}$ Zur Erklärung des Wachstums pro Kopf der Bevölkerung muss deshalb modellexogen ein nicht weiter erklärter technischer

57 Malthus 1798; Ricardo 1821.

58 Mill 1909.

59 Keynes in seiner Rede »An meine Enkel «, 1932.

60 Vgl. Aghion, Howitt 2009, S. 12 ff.

61 Solow 1968, S. 72. 
Fortschritt herangezogen werden, der die Produktivität von Arbeit und Kapital ständig steigert und so eine Zunahme der Wirtschaftsleistung ermöglicht. ${ }^{62}$

Das AK-Modell geht den ersten Schritt zur modellendogenen Erklärung von technischem Fortschritt dadurch, dass Kapital explizit als die Summe aus physischem Kapital und produktivitätssteigerndem Humankapital konzipiert ist. Für dieses Kapitalaggregat muss dann kein fallender Grenzertrag mehr angenommen werden, weil im Prozess der Kapitalakkumulation das Humankapital und damit auch der resultierende technische Fortschritt mitwachsen. Der Wachstumsmotor dieses Modells ist daher die Sparquote: Ersparnis ermöglicht die Finanzierung von technischem Fortschritt als Grundlage weiteren Wachstums. Die Entstehung von Humankapital und technischem Fortschritt wird jedoch auch in diesem Modell nicht explizit erklärt.

Mit dem Product-Variety Model gelang Romer die erste explizite Formalisierung des technischen Fortschritts, indem in die bis dato monolithische Struktur der modellierten Volkswirtschaft ein Forschungssektor eingeführt wurde. ${ }^{63}$ Die gesamtwirtschaftliche Produktionsfunktion ist dabei die Summe aus vielen einzelnen, produktbezogen formulierten Produktionsfunktionen. Die fallenden Grenzerträge der Kapitalverwertung werden in diesem Modell dadurch überwunden, dass als Ergebnis von Forschung und Entwicklung ständig neue Produkte entstehen. Die Rate, mit der die Produktvielfalt wächst, ist daher langfristig identisch zur Wachstumsrate des Sozialprodukts pro Kopf.

Die Unvollkommenheiten des Product-Variety Model - es kommen immer mehr Produkte hinzu, aber neuere Produkte verdrängen ältere nicht vom Markt; der Aufstieg neuer und der Untergang alter Firmen werden nicht modelliert - werden im Schumpeter'schen Wachstumsmodell ${ }^{64}$ überwunden. Es trägt der zentralen Idee des österreichisch-amerikanischen Nationalökonomen Joseph Schumpeter Rechnung, dass das Wesen des Kapitalismus in Innovationen und der »schöpferischen Zerstörung " vorhandener Prozesse und Produkte liege: In schumpeterianischen Modellen führen qualitätsverbessernde Innovationen zu neuen Produktvarianten, die ältere Produkte obsolet machen und vom Markt verdrängen. In jeder Industrie kann sich nur der Anbieter mit der jeweils führenden Technologie behaupten - bis er selbst vom nächsten Innovator überholt und vom Markt verdrängt wird. Das Wachstum der Gesamtwirtschaft resultiert folglich aus der Innovationsgeschwindigkeit - und dem damit verbundenen Eintritt und Austritt innovativer Firmen - in den einzelnen Branchen. Weil als Inputs für den branchenbezogenen Innovationsprozess nicht nur die Forschungs- und Entwicklungsausgaben des jeweiligen Unternehmers zählen, sondern auch der akkumulierte Bestand bisheriger Innovationen betrachtet wird, kann das Modell auch komplexe Innovationsdynamiken sowie Kontext- und Pfadabhängigkeiten abbilden, die für hochentwickelte Volkswirtschaften von Bedeutung sind.

62 Vgl. auch Pollitt et al. 2010.

63 Romer 1990.

64 Aghion, Howitt 1992, 1998.

Leviathan, 40. Jg., 2/2012 
In den Basisvarianten dieser vier Modelle spielen - wie bereits erwähnt - ökologische Restriktionen keine Rolle. Insbesondere wird nicht berücksichtigt, dass physisches Kapital notwendigerweise unter Nutzung von Material und Energie, also natürlicher Ressourcen hergestellt wird. ${ }^{65}$ Durch Berücksichtigung natürlicher Ressourcen als Input im Produktionsprozess können jedoch die Folgen ökologischer Restriktionen für den Verlauf und mögliche Grenzen des Wachstumsprozesses im Kontext dieser Modelle analysiert werden (siehe unten).

Als ein Zweig der Wirtschaftswissenschaft, der Konflikte zwischen Wirtschaft und Umwelt analysiert und Lösungen dafür sucht, entstand die Umwelt- und Ressourcenökonomie. Sie ist aus der neoklassischen Theorie hervorgegangen und integriert Umweltprobleme in deren Logik und Konzepte. ${ }^{66} \mathrm{Im}$ Kern geht es bei der Umweltund Ressourcenökonomie um die optimale Nutzung begrenzt vorhandener natürlicher Ressourcen ${ }^{67}$ und um die negativen externen Umwelteffekte wirtschaftlicher Aktivitäten.

Aus der Erkenntnis, dass Märkte beim Schutz natürlicher Gemeingüter (etwa sauberer Luft und sauberen Wassers) und bei der Vermeidung von externen Effekten versagen, wird die Notwendigkeit abgeleitet, durch ökonomische Instrumente der Umwelt »einen Preis zu geben « und das ökonomische Eigeninteresse der Verursacher von Umweltschäden nutzbar zu machen. Ein weiterer Ansatz aus der Umweltökonomie ist eine Verteilung der Eigentumsrechte, die zu einer optimalen Nutzung der Umwelt führen sollen. Wie die Neoklassik beruht die Umweltökonomie damit auf dem Ansatz des methodischen Individualismus, der wirtschaftliches Geschehen letztlich als Ergebnis der Handlungen der einzelnen, ihren jeweiligen Nutzen maximierenden Individuen deutet. ${ }^{68}$

Zur Frage ökologischer Grenzen des Wachstums positioniert sich die Umweltund Ressourcenökonomie nicht eindeutig. Eine wesentliche Kontroverse dreht sich um die Substituierbarkeit verschiedener Formen von Kapital. Grenzen des Wachstums existieren dann, wenn nichterneuerbare Ressourcen unverzichtbar für die Aufrechterhaltung des Wachstums, aber nicht durch menschengemachtes Kapital ${ }^{69}$ substituierbar sind. Traditionell herrscht in der Neoklassik jedoch Technologieoptimismus: Durch Effizienzsteigerungen, neue Technologien oder die Substitution natürlicher Ressourcen durch technisches Kapital können die Grenzen überwunden

65 Dazu kritisch z.B. Georgescu-Roegen 1975; Daly 1997; Ayres, Warr 2010; Scherhorn 2010.

66 Pollitt et al. 2010, S. 6.

67 Bereits in den 1970er Jahren: Solow 1974; Dasgupta, Heal 1979.

68 Weimann 1995, S. 170 ff.; Faber, Manstetten 2007, S. 350 ff.; Bergstrom, Randall 2010, S. $71 \mathrm{ff}$.

69 Menschengemachtes Kapital bezeichnet Produktionsmittel, die von Menschen hergestellt wurden, also etwa Geräte, Maschinen, Fabrikhallen, im Gegensatz zum von der Natur ohne Zutun des Menschen bereitgestellten natürlichen Kapital, etwa Boden, Wasser, Ökosysteme. 
werden. ${ }^{70}$ Eine zentrale Rolle in der Argumentation spielen hier sogenannte Backstopp-Technologien, die ein unbegrenzt nutzbares Substitut für eine begrenzte nichterneuerbare Ressource bereitstellen können. ${ }^{71}$ Dieser Glaube an vollständige Substituierbarkeit stellt allerdings auch die zentrale Schwachstelle der Umwelt- und Ressourcenökonomie dar, da sie physikalische Gesetze ignoriert und im Grunde das zu lösende Problem - die optimale Art des Umgangs mit begrenzten Ressourcen umgeht.

Ein illustratives Beispiel für dieses Defizit findet sich in dem aktuellen Lehrbuch »The Economics of Growth « ${ }^{72}$. Das Kapitel »Preserving the Environment «nimmt zunächst an, dass die Produktion eines bestimmten Guts die Umwelt belastet, also den »Kapitalstock « einer Umweltressource reduziert. Das Wachstum dieser »schmutzigen Produktion « (»dirty production «) unterliegt dementsprechend einer Geschwindigkeitsbegrenzung, die durch die Regenerationsrate der Umweltressource vorgegeben ist. Diese Begrenzung kann jedoch entweder durch unendlich steigerbare Innovation und Effizienzverbesserung aufgelöst werden ${ }^{73}$ oder durch die Annahme, dass durch technischen Fortschritt ein zweiter, äquivalenter Produktionsprozesses gefunden werden kann, der den Bestand der Umweltressource nicht beeinflusst (»clean production «). Die politische Aufgabe besteht dann nur noch darin, durch angemessene Besteuerung einen Wechsel von der "dirty production « hin zur »clean production « zu bewerkstelligen. Das Ergebnis dieser Übung ist: Das Wachstum kann ungehindert fortgesetzt werden.

Aus Sicht der Umweltwissenschaft sind derartige Lösungskonzepte der Problematik nicht angemessen und unterkomplex, weil eine entscheidende Restriktion letztlich per Annahme aufgehoben wird. Wesentliche Fragen werden ausgeblendet, die sich nur empirisch beantworten lassen: Inwieweit kann Wertschöpfung wirklich von einer materiellen Basis unabhängig gemacht werden? Existieren erneuerbare Ressourcen, die unbegrenzt nutzbar sind? Oder gehört die Vorstellung einer vollständig »sauberen " Produktion nicht vielmehr ins Reich der Utopie? ${ }^{74}$ In der Realität dürfte es in den meisten Fällen eher darum gehen, stärker umweltbelastende durch weniger belastende Produktionsweisen zu ersetzen. Dadurch aber würde die "Geschwindigkeitsbegrenzung « für den Wachstumsprozess bestenfalls gelockert, nicht aber aufgehoben.

Robert Solow und Joseph Stiglitz haben zwar Ende der 1990er Jahre in einer Antwort auf eine heftige Kritik des ökologischen Ökonomen Herman Daly bestimmte Grenzen der Substituierbarkeit eingestanden. ${ }^{75}$ Unter anderem stimmten sie zu, dass eine vollständige Substitution von natürlichem durch menschengemachtes Kapital nicht möglich ist, da physisches Kapital immer den Abbau natürlicher

70 Vgl. z.B. Stiglitz 1974; Solow, Wan 1974.

71 Vgl. z.B. Solow 1974; Dujmovits 2009.

72 Aghion, Howitt 2009.

73 Ebd., S. 379 ff.

74 Huesemann 2003.

75 Daly 1997; Solow 1997; Stiglitz 1997.

Leviathan, 40. Jg., 2/2012 
Ressourcen voraussetzt. Solow und Stiglitz erkennen zudem an, dass der Grad der Substituierbarkeit eine empirische Frage ist, die mehr Aufmerksamkeit erfordert. Dennoch werden diese Erkenntnisse im wirtschaftswissenschaftlichen »Mainstream « bisher kaum umgesetzt. Eine angemessene Auseinandersetzung mit der Bedeutung ökologischer Grenzen für wirtschaftliches Wachstum findet häufig nicht statt. Selbst in dem Standardlehrbuch »Introduction to Modern Economic Growth " von Daron Acemoglu ${ }^{76}$ gibt es trotz eines Umfangs von knapp 1.000 Seiten keinen einzigen Verweis auf ökologische Restriktionen und physische Grenzen des Wachstums. Sofern Umweltfragen überhaupt einbezogen werden, steht nach wie vor im Vordergrund, welche Risiken für die Wirtschaft in der Begrenztheit einzelner natürlicher Ressourcen liegen und wie diese überwunden werden können. Das $\mathrm{Zu}$ sammenspiel mehrerer ökologischer Grenzen, die Gefährdung ökosystemarer Funktionszusammenhänge durch Verschmutzung und Übernutzung erneuerbarer Ressourcen, die Möglichkeit nichtlinearen Verhaltens von natürlichen Systemen und Nutzungskonflikte werden nicht berücksichtigt.

\section{2 Ökologische Ökonomie}

Im Gegensatz zur Umwelt- und Ressourcenökonomie macht die Ökologische Ökonomie die Begrenzungen des Umweltsystems zum Ausgangspunkt der Theoriebildung. Damit beruht sie auf einem grundlegend anderen Verständnis der Wechselwirkungen zwischen Umwelt und Wirtschaft. Ökologische Ökonomen stellen viele Annahmen der traditionellen wirtschaftswissenschaftlichen Theorien, insbesondere der Neoklassik, radikal in Frage und fordern teilweise eine tiefgehende Überarbeitung des ökonomischen Theoriegebäudes. ${ }^{77}$

Die Ökologische Ökonomie orientiert sich am Leitbild der nachhaltigen Entwicklung als einem normativen Rahmen für wirtschaftliches Handeln. Die bekannte Definition des Begriffs "nachhaltige Entwicklung « (Sustainable Development) im Bericht der Brundtland-Kommission ${ }^{78}$ stellt die Befriedigung der Bedürfnisse der Menschen in den Vordergrund, spiegelt aber gleichzeitig die Erkenntnis wider, dass menschliches Handeln potenziell die Möglichkeiten für die Bedürfnisbefriedigung anderer Menschen gefährden bzw. die Befriedigung von menschlichen Bedürfnisse an absolute Grenzen stoßen kann. ${ }^{79}$

Der Erhalt der natürlichen Lebensgrundlagen leitet sich daraus als zentrale Voraussetzung einer nachhaltigen Entwicklung ab. Da die Tragfähigkeit der natürlichen Systeme begrenzt ist (vgl. Abschnitt 2.2), müssen Wirtschaftssysteme und Gesellschaften letztendlich als in dieses ökologische System eingebettete Subsysteme ver-

76 Acemoglu 2009.

77 Vgl. Common, Stagl 2005; Pollitt et al. 2010.

78 »Nachhaltige Entwicklung ist eine Entwicklung, die den Bedürfnissen der heutigen Generation entspricht, ohne die Möglichkeiten künftiger Generationen zu gefährden, ihre eigenen Bedürfnisse zu befriedigen und ihren Lebensstil zu wählen. «; Hauff 1987, S. 46.

79 WCED 1987. 
standen werden. ${ }^{80}$ Als solche sind sie den Gesetzen der Thermodynamik unterworfen. Um Strukturen zu schaffen, beispielsweise in der wirtschaftlichen Produktion, muss nutzbare Energie eingesetzt werden; dabei entsteht Entropie (nicht nutzbare Energie). Ökonomische Wertschöpfung ist notwendigerweise mit der Nutzung der Umwelt - als Rohstoffquelle und als Senke für Schadstoffe - verbunden.

Entsprechend heftig kritisieren Vertreter der Ökologischen Ökonomie die Naturvergessenheit der Hauptströmung in der Wirtschaftswissenschaft, die den Menschen letztendlich »zum alleinigen Produzenten und Schöpfer der Wirtschaft « ${ }^{81}$ erklärt. Der Mathematiker und Ökonom Nicholas Georgescu-Roegen wies früh schon auf die Vernachlässigung thermodynamischer Gesetze in der Ökonomie hin. Er sah im ökonomischen Kreislaufmodell, welches in seiner Grundform ein geschlossenes System aus Güter- und Geldkreislauf zwischen Unternehmen und Haushalten darstellt und die notwendigen Inputs aus der Natur in diesen Wirtschaftskreislauf ignoriert, die »Erbsünde « der modernen Nationalökonomie, da sie suggeriere, die Wirtschaft könne aus sich selbst heraus wachsen und sich erhalten. ${ }^{82}$ Die Vorstellung eines begrenzten Umweltraums, in dem sich menschliches Wirtschaften abspielt, zwingt zu einer Neubewertung von wirtschaftlichen Zielen und Maßstäben. Nach Kenneth Boulding kann es in der - bis auf die Energiezufuhr von der Sonne - geschlossenen Welt, in der die Menschheit sich befindet, nicht das Ziel sein, den Durchfluss von Material in Form von Produktion und Konsum immer weiter zu steigern - vielmehr sollte dieser minimiert werden. Der Erfolg des Wirtschaftens in einer solchen Ökonomie misst sich nicht an Flussgrößen, sondern an Bestandsgrößen. Maßstab für den Erfolg ist die Größe, Qualität und Komplexität des gesamten Kapitalbestands, zu dem Boulding neben dem natürlichen Kapital auch die in menschlichem Wissen und menschlicher Kultur eingebettete Information zählt. ${ }^{83}$

Herman E. Daly formalisiert diese Vorstellung in seinem Modell einer SteadyState-Ökonomie. Darin ist die Ökonomie Subsystem einer begrenzten Biosphäre und kann sich in ihrer materiellen Dimension nicht unendlich ausdehnen. Es muss deshalb eine optimale Größe der Gesamtwirtschaft in Relation zum Ökosystem geben. Der Bestand an physischem Kapital ist dann konstant und nur so groß, dass er mit einer niedrigen Rate des Materialdurchlaufs aufrechterhalten werden kann, die innerhalb der Regenerations- und Assimilationsfähigkeit des Ökosystems liegt. ${ }^{84}$

Die Ökologische Ökonomie hat sich seit ihren Anfängen als eine eigene - wenn auch kleine - Richtung der Wirtschaftswissenschaft etabliert. Seit 1989 dokumentiert die Zeitschrift »Ecological Economics « die Entwicklungen der Disziplin und das anhaltende wissenschaftliche Interesse an ihren Inhalten. Dennoch ist sie eine Nischendisziplin geblieben. In der aktuelleren Literatur sind bisher nur konzeptionelle Ansätze einer ökologischen Makroökonomie oder Forderungen nach ihrer

80 Common, Stagl 2005, S. 87; Hall et al. 2001, S. 665.

81 Binswanger 2010, S. 73.

82 Georgescu-Roegen 1971.

83 Boulding 1966.

84 Daly 1996.

Leviathan, 40. Jg., 2/2012 
Entwicklung zu erkennen. Baumgärtner und Quaas beispielsweise formulieren Prinzipien einer sustainability economics, die die Beziehung zwischen Mensch und Natur zum Gegenstand der Analyse macht und auf eine langfristige Zukunft hin orientiert ist. Ihre normative Begründung liegt in der Idee der Gerechtigkeit als zentralem Element der Nachhaltigkeit, sowohl zwischen Menschen heutiger und zukünftiger Generationen als auch zwischen Mensch und Natur. ${ }^{85}$ Der britische Ökonom Tim Jackson skizziert eine ökologische Makroökonomie, die unabhängig vom Wachstum funktionieren soll und auf einem differenzierteren Umgang mit dem Begriff des Kapitals beruht. ${ }^{86}$ Peter Victor nutzt vereinfachte Computersimulationen auf der Basis eines konventionellen Wirtschaftsmodells, um am Beispiel Kanadas auszuloten, unter welchen Bedingungen eine Volkswirtschaft ohne Wachstum funktionieren und somit absolute physische Grenzen einhalten könnte. ${ }^{87}$

\subsection{Zwischenfazit}

Eine ökologisch sensibilisierte Ökonomietheorie würde grundsätzlich die Tatsache berücksichtigen, dass Produktion ohne Energie- und Materialeinsatz nicht möglich ist. Sie müsste zugleich als wesentliches Ziel des Wirtschaftens definieren, dass ökologische Grenzen nicht überschritten werden. Es ist anzunehmen, dass sich der Stellenwert des Wirtschaftswachstums in einer derartigen Ökonomietheorie verändern würde. Allerdings gibt die Hauptströmung der Wirtschaftswissenschaft bislang keine befriedigende Antwort auf die Frage, wie sich ökologische Grenzen - sei es durch ihre Überschreitung oder durch vorausschauende Begrenzung von Ressourcen- und Umweltverbrauch - auf die gesamtwirtschaftliche Aktivität auswirken und wie damit verbundenen Risiken und Problemen vorgebeugt werden kann.

Vielmehr spiegelt sich das Wachstumsdilemma in dem weitgehend unverbundenen Nebeneinander von Mainstream-Ökonomie und Ökologischer Ökonomie. Während der Mainstream in der Regel weiterhin auf die Maximierung des Wachstums unter Vernachlässigung ökologischer Nebenbedingungen setzt, stellt die Ökologische Ökonomie zwar die ökologischen Restriktionen in den Mittelpunkt, liefert aber bislang keine schlüssige und umfassende Theorie für ein nachhaltiges, möglicherweise auch wachstumsunabhängiges Wirtschaften, das Stabilität und Funktionsfähigkeit der Systeme sowie eine hohe Lebensqualität garantiert.

Um das Wachstumsdilemma angemessen untersuchen und Auswege finden zu können, muss sich das Nachdenken über Wirtschaft und die Formalisierung dieses Nachdenkens durch die Theorie verändern. Dafür sollten der wirtschaftswissenschaftliche Mainstream und die ökologisch orientierten Ökonomen stärker aufeinander zugehen und sich in der Erarbeitung einer integrierten Theorie engagieren. Als Ausgangspunkt erscheinen Bestrebungen vielversprechend, einerseits eine umfassende Produktionsfunktion zu modellieren, die Naturkapital und Sozialkapital den gleichen Rang zuweist wie physischem und finanziellem Kapital sowie Human-

85 Baumgärtner, Quaas 2010.

86 Jackson 2009, S. 209 ff.

87 Victor 2008. 
kapital, ${ }^{88}$ und andererseits eine Nutzenfunktion der Individuen anzunehmen, die neben Einkommen auch soziales und natürliches Kapital als Argument berücksichtigt. Modelle auf der Basis einer derart integrierten Sicht könnten dann die Identifizierung und Analyse der entscheidenden Wechselwirkungen und Spannungsverhältnisse zwischen den verschiedenen Kapitalformen im Wachstumsprozess erlauben. ${ }^{89}$

Auf diese Weise könnte das bewährte Instrumentarium der Wirtschaftswissenschaft in der Breite an Erkenntnisse aus der Ökologie und neue Problemgewichtungen angepasst werden. Durch die Auseinandersetzung mit neuen Herausforderungen - etwa mit der Frage, wie auch bei sinkenden Wachstumsraten oder bei ausbleibendem Wachstum Stabilität und Wohlstand in modernen Marktwirtschaften erhalten werden können - kann die Wirtschaftswissenschaft neues Profil gewinnen und ihre Rolle für die Politikberatung auch unter veränderten Rahmenbedingungen erhalten.

\section{Plädoyer für eine ökologische Schuldenbremse}

Für die Politik stellt sich die Frage, wie sie - trotz fehlender ausgereifter Konzepte und Theorien - konstruktiv mit dem Wachstumsdilemma umgehen kann. Die ökologischen Probleme gezielt über eine Beschränkung oder Lenkung des Wachstums lösen zu wollen, ist unseres Erachtens weder realistisch noch zielführend. Zwar besteht eindeutig ein Zusammenhang zwischen Wachstum und Umweltbelastung, umgekehrt folgt daraus aber nicht, dass über das Wachstum der Zustand der Umwelt direkt beeinflusst werden könnte. Vielmehr bestünde das große Risiko, wirtschaftliche Verwerfungen hervorzurufen, ohne die ökologischen Probleme zu lösen - abgesehen davon, dass eine derartige Strategie politisch keinerlei Aussichten auf Erfolg haben würde. Stattdessen sollten deshalb die ökologischen Belastungsgrenzen selbst zum Ansatzpunkt werden. Wird die Abhängigkeit menschlichen Lebens und Wirtschaftens von den natürlichen Lebensgrundlagen sowie deren Begrenztheit anerkannt, muss die Einhaltung der ökologischen Grenzen zu einer allgemeinen Rahmenbedingung für wirtschaftliche Aktivitäten werden. Die Regenerationsfähigkeit von Ökosystemen und erneuerbaren Ressourcen wird zum entscheidenden Kriterium für ihre Nutzung oder ihre Belastung mit Schadstoffen und Abfällen, wenn ökologisches Kapital für die Zukunft erhalten werden soll. Dies muss einen Paradigmenwechsel nach sich ziehen: von relativ zur Wirtschaftsleistung formulierten Umweltzielen wie der Steigerung der Ressourcenproduktivität oder der Erhöhung der Energieeffizienz hin zu absoluten Mindeststandards und Obergrenzen.

Im Bereich der Finanzpolitik ist es in Deutschland mit der sogenannten Schuldenbremse für Bund und Länder gelungen, eine stärkere Orientierung auf Langfristigkeit im Grundgesetz zu verankern. Selbst wenn die tatsächliche Wirkung des Instruments noch abzuwarten bleibt und viel vom verantwortungsvollen Umgang da-

88 Vgl. Bergstrom, Randall 2010, S. 25 f.

89 Vgl. Roseta-Palma et al. 2010.

Leviathan, 40. Jg., 2/2012 
mit abhängt - wie die unterschiedlichen Erfahrungen mit ähnlichen Instrumenten etwa in der Schweiz und den USA zeigen -, ist ein wesentlicher Schritt zu mehr finanzpolitischer Nachhaltigkeit erfolgt.

Für die ökologische Problematik ist ein Instrumentarium nötig, welches eine Begrenzung ebenso stringent einfordert und durchsetzt. Die Parallelen sind augenfällig, wenn man die Problemstruktur in der Kapitalmetaphorik betrachtet: Der Versuchung, vom Kapitalstock der Umwelt zugunsten kurzfristiger Vorteile zu zehren und dadurch die ökologischen Schulden zulasten künftiger Generationen immer weiter zu erhöhen, können die beteiligten Akteure häufig nicht aus eigener Kraft widerstehen. Deshalb ist eine Bindung nötig, die einen institutionellen Schutz vor dieser Versuchung bietet und somit auch die biophysischen Grundlagen für die Ökonomie der Zukunft sichert: eine ökologische Schuldenbremse.

Die finanzpolitische Schuldenbremse kann dabei Anregungen geben, welche Elemente notwendig sind, um ein derartiges Instrument wirksam auszugestalten. So sollte auch die "ökologische Schuldenbremse " in der Verfassung verankert sein, einzuhaltende Zielwerte vorgeben, Übergangsfristen formulieren und prozedurale Regeln und Institutionen schaffen, die die Umsetzung überwachen und gegebenenfalls auch durchsetzen können.

Dabei sind allerdings mit der Einrichtung einer Schuldenbremse in der Ökologie noch ungleich größere Herausforderungen verbunden als in der Finanzpolitik. Scheitern kann sie bereits bei der Festlegung der Obergrenzen: Die Umweltwissenschaft kann die ökologischen Belastungsgrenzen nur in den seltensten Fällen genau quantifizieren und gibt aufgrund der Komplexität der Zusammenhänge oft nur entsprechende Korridore an. Die Unsicherheit in den naturwissenschaftlichen Erkenntnissen kann politische Untätigkeit jedoch nicht rechtfertigen: Es gibt ausreichend Hinweise darauf, dass sich die Umweltbelastungen bereits im roten Bereich befinden oder sich rasch auf diesen zubewegen. Das Vorsorgeprinzip verlangt unter diesen Umständen ein Handeln auch unter Unsicherheit. Die Festlegung von politischen Zielwerten erfordert dann allerdings normative Entscheidungen: Welche Veränderungen in den Umweltbedingungen sind wir bereit, auch im Namen zukünftiger Generationen zu akzeptieren, welche Risiken bereit, für die Nachwelt einzugehen? Begrenzungen können also nicht einfach aus der Naturwissenschaft "abgerufen « werden, sondern müssen in gesellschaftlichen Prozessen als möglichst breiter und belastbarer Konsens erarbeitet werden.

Zudem müsste eine ökologische Schuldenbremse - anders als die finanzpolitische - letztendlich multidimensional konstruiert sein. Es geht nicht nur darum, eine einzelne begrenzt vorhandene Ressource zu substituieren, sondern darum, verschiedene ökologische Belastungen gleichzeitig in Grenzen zu halten und zu verhindern, dass durch die Lösung des einen Problems ein anderes geschaffen wird.

Bereits die finanzpolitische Schuldenbremse betrifft mit Bund und Ländern eine Vielzahl von Akteuren, deren Verhalten koordiniert bzw. kontrolliert werden muss, damit das Instrument die gewünschte Wirkung erzielt. Bei der Umweltbelastung ist zudem das Handeln von Unternehmen und privaten Haushalten relevant - es sind Mechanismen wie etwa Emissionshandelssysteme notwendig, die das Verhalten großer Akteursgruppen oder ganzer Gesellschaften auf Umweltziele hin ausrichten 
können. Um Erfindungsreichtum und Innovationskraft von Gesellschaft und Wirtschaft möglichst stark zu beflügeln statt sie zu begrenzen, sollten dabei zwar Grenzund Zielwerte als Leitplanken vorgegeben werden, nicht jedoch im Detail die zu ihrer Berücksichtigung und Erreichung einzusetzenden Technologien. Nur auf diese Weise kann die ökologische Modernisierung der Wirtschaft marktwirtschaftlich und kosteneffizient erreicht werden, und nur so besteht die Chance, trotz zu erwartender Interessenskonflikte eine grundlegende Akzeptanz in Wirtschaft und Gesellschaft zu erreichen.

Und schließlich führt die internationale Dimension von Umweltproblemen immer wieder in die Problemstruktur eines Gefangenendilemmas, was politisches Handeln auf nationaler Ebene erschwert und internationale Abstimmung und Kooperation erfordert. Wie kompliziert die Interessengefüge und nahezu unlösbar die Konflikte zwischen den Staaten sind, illustriert der Prozess der internationalen Klimaverhandlungen. ${ }^{90}$

Gerade angesichts der Komplexität der Herausforderung kann die Politik nicht aus ihrer Verantwortung entlassen werden. Ansatzpunkte für eine ökologische Schuldenbremse existieren bereits in Form politisch gesetzter Umweltziele auf verschiedenen Ebenen und für unterschiedliche Bereiche - von den Indikatoren der deutschen Nachhaltigkeitsstrategie über Ziele für Emissionsreduktion und Energieeffizienz auf europäischer Ebene bis hin zu den international vereinbarten Zielen im Kyoto-Protokoll oder in der Biodiversitätskonvention. Wie in der Finanzpolitik ist jedoch ausschlaggebend, dass es nicht bei der Formulierung von Zielen bleibt, sondern dass Mechanismen geschaffen werden, die konkrete einzelne Entscheidungen in der Gegenwart im Sinne dieser Ziele beeinflussen können. Eine ökologische Schuldenbremse, die Umweltziele gesetzlich verankert und Institutionen und Verfahren zu ihrer Umsetzung schafft bzw. stärkt, muss deshalb Schritt für Schritt aufgebaut werden.

Die Vorteile, die eine ökologische Schuldenbremse mit sich bringen würde, werden deutlich und kommunizierbar, wenn ein neues, umfassendes Konzept von Wohlstand zugrunde gelegt wird. Die Politik sollte sich orientieren an einem Wohlstandsbegriff, der nicht wie das BIP den »Umsatz« einer Volkswirtschaft, sondern den Wert ihres Vermögens misst. Wie auch immer dieser neue Wohlstandsbegriff operationalisiert wird, das neue Messsystem müsste Zielkonflikte zwischen einer Steigerung des BIP und einem Verlust an Natur widerspiegeln. Eine Erhaltung oder auch Vermehrung des so umfassend verstandenen Wohlstands müsste dann im Konfliktfall Priorität vor der Steigerung des BIP erhalten. Damit würde das Wachstum der Wirtschaft nicht grundsätzlich ausgeschlossen. Zwar könnten bestimmte Ausprägungen des heutigen Wachstums, die auf möglichst kurze Produktlebenszyklen und immer weiter steigenden Konsum materieller Güter setzen, durch derart gesetzte Rahmenbedingungen unwirtschaftlich werden; innerhalb der definierten ökologischen Grenzen könnte sich die Wirtschaft insgesamt jedoch weiter entwickeln und wachsen, sofern das Wachstum aus Quellen gespeist wird, die nicht mit diesen Grenzen in Konflikt geraten. Innovationen würden in entsprechende Bahnen 
gelenkt. Angesichts der noch bestehenden großen Potenziale für eine Entkopplung der Wirtschaftsleistung vom Umweltverbrauch und der wirtschaftlichen Chancen, die ein effizienter und nachhaltiger Umgang mit natürlichen Ressourcen birgt, wäre weiteres Wachstum zumindest in der näheren Zukunft dann möglich. Die Höhe der Wachstumsraten würde aber in der politischen und gesellschaftlichen Diskussion an Bedeutung verlieren, denn die ökologische Schuldenbremse trüge zu einer Verschiebung der Aufmerksamkeit bei: von der Maximierung des Wachstums hin zur Optimierung des Wohlstands.

\section{Literatur}

Acemoglu, Daron 2009. Introduction to modern economic growth. Princeton: Princeton University Press.

Aghion, Philippe; Howitt, Peter 1992. "A model of growth through creative destruction «, in Econometrica 60, 2, S. 323-351.

Aghion, Philippe; Howitt, Peter 1998. Endogenous growth theory. Cambridge, London: MIT Press.

Aghion, Philippe; Howitt, Peter 2009. The economics of growth. Cambridge, London: MIT Press.

Ayres, Robert U.; Warr, Benjamin 2010. The economic growth engine. How energy and work drive material prosperity. Cheltenham: Edward Elgar.

Barker, Terry; Dagoumas, Athanasios; Rubin, Jonathan 2009. »The macroeconomic rebound effect and the world economy ", in Energy Efficiency 2, 4, S. 411-427.

Baumgärtner, Stefan; Quaas, Martin 2010. »What is sustainability economics? ", in Ecological Economics 69, S. 445-450.

Bergstrom, John C.; Randall, Alan 2010. Resource economics. An economic approach to natural resource and environmental policy. 3. Aufl. Cheltenham: Edward Elgar.

BGR (Bundesanstalt für Geowissenschaften und Rohstoffe) 2009. Energierohstoffe 2009. Reserven, Ressourcen, Verfügbarkeit. Hannover: BGR.

Binswanger, Hans Christoph 2010. Vorwärts zur Mäßigung. Perspektiven einer nachhaltigen Wirtschaft. 2. Aufl. Hamburg: Murmann.

BMU (Bundesministerium für Umwelt Naturschutz und Reaktorsicherheit); UBA (Umweltbundesamt) 2010. Umweltbewusstsein in Deutschland 2010. Ergebnisse einer repräsentativen Bevölkerungsumfrage. Berlin, Dessau-Roßlau: BMU, UBA.

Börsch-Supan, Axel 2011. »Unsere gewonnenen Jahre«, in Frankfurter Allgemeine Zeitung, 25. Februar 2011, S. 11.

Booth, Douglas E. 2004. Hooked on growth. Economic addictions and the environment. Lanham: Rowman \& Littlefield.

Boulding, Kenneth E. 1966. "The economics of the coming spaceship earth ", in Environmental quality in a growing economy: essays from the Sixth RFF Forum, hrsg. v. Jarrett, Henry, S. 3-14. Baltimore: Johns Hopkins Press.

CBD (Convention on Biological Diversity) 2010. Global biodiversity outlook 3. Executive summary. Montréal: Secretariat of the Convention on Biological Diversity.

Cole, Hugh S. D. et al. Hrsg. 1973. Thinking about the future. A critique of the limits to growth. London: Chatto \& Windus.

Common, Michael S.; Stagl, Sigrid 2005. Ecological economics: an introduction. Cambridge: Cambridge University Press.

Coyle, Diane 2011: The economics of enough. How to run the economy as if the future matters. Princeton: Princeton University Press.

Daly, Herman E. 1996. Beyond growth. The economics of sustainable development. Boston: Beacon Press.

Daly, Herman E. 1997. »Georgescu-Roegen versus Solow/Stiglitz «, in Ecological Economics 22, S. 261-266. 
Daly, Herman E. 2008. »Economics blind spot is a desaster for the planet «, in New Scientist, 15. Oktober 2008.

Dasgupta, Partha; Heal, Geoffrey M. 1979. Economic theory and exhaustible resources. Cambridge: Cambridge University Press.

DeLong, J. Bradfold 2006. »Growth is good «, in Harvard Magazine, January-February 2006. http://harvardmagazine.com/2006/01/growth-is-good.html (Zugriff vom 04.04.2012).

Diefenbacher, Hans; Zieschank, Roland 2009. Wohlfahrtsmessung in Deutschland. Ein Vorschlag für einen nationalen Wohlfahrtsindex. Dessau-Roßlau: Umweltbundesamt.

Dujmovits, Rudolf 2009. Nicht erneuerbare Ressourcen, Grenzen des Wachstums und Fallstricke des technologischen Optimismus. Working Paper No. 2009-1. http://www.uni-graz.at/ fwiwww/2010/dt/forsch/wp/wp2009-1.pdf (Zugriff vom 04.04.2012).

ECF (European Climate Foundation) et al. 2010. Roadmap 2050: a practical guide to a prosperous, low-carbon Europe. Vol. 1: Technical and economic analysis. Den Haag: ECF.

Edenhofer, Ottmar et al. 2010. "The economics of low stabilization: model comparison of mitigation strategies and costs ", in The Energy Journal 31, Special Issue 1, S. 11-48.

Enderlein, Henrik 2010. »Wenn der Kuchen nicht mehr größer wird «, in Berliner Republik 2/2010. http://www.b-republik.de/aktuelle-ausgabe/wenn-der-kuchen-nicht-mehr-groesserwird (Zugriff vom 04.04.2012).

EREC (European Renewable Energy Council); Greenpeace International 2010. Energy [r]evolution. A sustainable world energy outlook. Brüssel, Amsterdam: EREC, Greenpeace International.

EREC 2010. RE-thinking 2050. A 100\% renewable energy vision for the European Union. Brüssel: EREC.

Faber, Malte; Manstetten, Reiner 2007. Was ist Wirtschaft? Von der politischen Ökonomie zur ökologischen Ökonomie. Freiburg, München: Alber.

Fitoussi, Jean-Paul; Laurent, Éloi 2008. La nouvelle écologie politique. Économie et développement humain. Paris: Éditions du Seuil et La République des Idées.

Flipo, Fabrice; Schneider, Francois 2008. Proceedings of the first international conference on economic de-growth for ecological sustainability and social equity. Paris, 18.-19. April 2008. http://events.it-sudparis.eu/degrowthconference/themes/ (Zugriff vom 04.04.2012).

FoEE (Friends of the Earth Europe); SEI (Stockholm Environment Institute) 2009. The 40\% study. Mobilising Europe to achieve climate justice. Brüssel, Stockholm: FoEE, SEI.

Friedman, Benjamin M. 2005. »The moral case for growth «, in The International Economy 40, S. 40-45.

Frondel, Manuel; Peters, Jörg; Vance, Colin 2008. "Identifying the rebound: evidence from a German household panel ", in Energy Journal 29, 4, S. 145-164.

Frondel, Manuel; Schmidt, Christoph M. 2004. »Facing the truth about separability: nothing works without energy", in Ecological Economics 51, 3-4, S. 217-223.

Frondel, Manuel; Schmidt, Christoph M. 2007. Von der baldigen Erschöpfung der Rohstoffe und anderen Märchen. RWI Positionen 19. Essen: RWI.

Gebhardt, Heinz; Kambeck, Rainer; Schmidt, Christoph M. 2009. Keine Steuererhöhungen! RWI Positionen 34. Essen: RWI.

Georgescu-Roegen, Nicholas 1971. The entropy law and economic process. Cambridge: Harvard University Press.

Georgescu-Roegen, Nicholas 1975. "Energy and economic myths «, in Southern Economic Journal 41, 3, S. 347-381.

Hall, Charles et al. 2001. "The need to reintegrate the natural sciences with economics ", in BioScience 51, 8, S. 663-673.

Hauff, Volker. Hrsg. 1987. Unsere gemeinsame Zukunft - Der Brundtland-Bericht der Weltkommission für Umwelt und Entwicklung. Greven: Eggenkamp.

Helm, Dieter; Smale, Robin; Phillips, Jonathan 2007. Too good to be true? The UK's climate change record. http://www.dieterhelm.co.uk/sites/default/files/Carbon_record_2007_1.pdf (Zugriff vom 04.04.2012).

Holm, Stig-Olof; Englund, Göran 2009. »Increased ecoefficiency and gross rebound effect: Evidence from USA and six European countries 1960-2002 ", in Ecological Economics 68, 3, S. 879-887.

Leviathan, 40. Jg., 2/2012 
Huesemann, Michael H. 2003. »The limits of technological solutions to sustainable development ", in Clean Technologies and Environmental Policy 5, S. 21-34.

IPCC (Intergovernmental Panel on Climate Change) 2007 a. Climate change 2007: impacts, adaptation and vulnerability. Cambridge: Cambridge University Press.

IPCC 2007 b. Climate change 2007: the physical science basis. Cambridge: Cambridge University Press.

Jackson, Tim 2009. Prosperity without growth. Economics for a finite planet. London: Earthscan.

Kavalov, Boyan; Peteves, Stathis D. 2007. The future of coal. Luxembourg: Europäische Kommission, Generaldirektion Joint Research Centre, Institute for Energy.

Lenton, Timothy M. et al. 2008. »Inaugural article: tipping elements in the Earth's climate system ", in Proceedings of the National Academy of Sciences 105, 6, S. 1786-1793.

Lowe, Adolph 1965. Politische Ökonomik. Frankfurt a. M.: Europäische Verlagsanstalt.

Malthus, Robert 1798. An essay on the principle of population. London: J. Johnson, in St. Paul's Church-yard.

MEA (Mannheim Research Institute for the Economics of Aging) 2007. Das falsche Nullsummenspiel mit der Arbeitszeit. MEA Policy Brief No. 3. Mannheim: MEA.

Meadows, Dennis et al. 1972. Die Grenzen des Wachstums. Bericht des Club of Rome zur Lage der Menschheit. Stuttgart: Deutsche Verlags-Anstalt.

Meadows, Donella; Randers, Jørgen; Meadows, Dennis 2006. Grenzen des Wachstums - Das 30-Jahre-Update. 2. Aufl. Stuttgart: Hirzel.

Miegel, Meinhard 2010. Exit. Wohlstand ohne Wachstum. 3. Aufl. Berlin: Propyläen.

Mill, John S. 1909. Principles of political economy, with some of their applications to social philosophy. Edited by William J. Ashley based on Mill's 7th edition. London: Longmans, Green and Co.

Millennium Ecosystem Assessment 2005. Ecosystems and human well-being: synthesis. Washington: Island Press.

Nässén, Jonas; Larsson, Jörgen; Holmberg, John 2009. »The effect of work hours on energy use. A micro-analysis of time and income effecs ", in Act! Innovate! Deliver! Reducing energy demand sustainably. ECEEE 2009 summer study, hrsg. v. ECEEE (European Council for an Energy Efficient Economy), S. 1800-1809. Stockholm: ECEEE.

OECD (Organisation for Economic Co-operation and Development) 2010. Interim report of the green growth strategy: implementing our commitment for a sustainable future. Paris: OECD.

Öko-Institut; Prognos AG 2009. Modell Deutschland - Klimaschutz bis 2050: Vom Ziel her denken. Endbericht. Berlin, Basel: Öko-Institut, Prognos.

Paech, Niko 2009 a. »Die Postwachstumsökonomie - ein Vademecum «, in Zeitschrift für Sozialökonomie 160-161, S. 28-31.

Paech, Niko 2009 b. "Wachstum >light ? Qualitatives Wachstum ist eine Utopie«, in Wissenschaft und Umwelt Interdisziplinär 13/2009, S. 84-93.

Paqué, Karl-Heinz 2010. Wachstum! Die Zukunft des globalen Kapitalismus. München: Carl Hanser Verlag.

Phelps, Edmund S. 2007. "Macroeconomics for a modern economy ", in American Economic Review 97, 3, S. 543-561.

Pollitt, Hector et al. 2010. A scoping study on the macroeconomic view of sustainability. Final Report for the European Commission, DG Environment. Cambridge, Wien: Cambridge Econometrics, Sustainable Europe Research Institute.

PwC (PricewaterhouseCoopers) et al. 2010. 100\% renewable electricity. A roadmap to 2050 for Europe and North Africa. London et al.: PwC et al.

Raffelhüschen, Bernd; Moog, Stefan 2010. Ehrbarer Staat? Die Generationenbilanz. Update 2010: Handlungsoptionen der Gesundheitspolitik. Argumente zu Marktwirtschaft und Politik, Nr. 111. Berlin: Stiftung Marktwirtschaft.

Reuter, Norbert 2010. »Der Arbeitsmarkt im Spannungsfeld von Wachstum, Ökologie und Verteilung ", in Postwachstumsgesellschaft. Konzepte für die Zukunft, hrsg. v. Seidl, Irmi; Zahrnt, Angelika, S. 85-102. Marburg: Metropolis.

Ricardo, David 1821. On the principles of political economy and taxation. 3. Aufl. London: John Murray. 
Rockström, Johan et al. 2009. "A safe operating space for humanity «, in Nature 461, S. 472-475.

Romer, Paul M. 1990. "Endogenous technological change ", in The Journal of Political Economy 98, 5, Part 2, S. 71-102.

Roseta-Palma, Catarina; Ferreira-Lopes, Alexandra; Sequeira, Tiago N. 2010. »Externalities in an endogenous growth model with social and natural capital «, in Ecological Economics 69, S. 603-612.

RWI (Rheinisch-Westfälisches Institut für Wirtschaftsforschung) 2010. Ermittlung der Konjunkturkomponenten für die Länderhaushalte zur Umsetzung der in der Föderalismuskommission II vereinbarten Verschuldungsbegrenzung. Endbericht zum Forschungsauftrag des Bundesministeriums der Finanzen. Essen: RWI.

Sachs, Wolfgang; Santarius, Tilman 2005. Fair Future. Begrenzte Ressourcen und Globale Gerechtigkeit. Ein Report. Wuppertal: Wuppertal-Institut für Klima, Umwelt, Energie.

Sachs, Wolfgang 2010. »Anders! Weniger! Besser! Konturen eines zukunftsfähigen Wirtschaftsmodells", in Politische Ökologie 27, 118, S. 37-40.

Scherhorn, Gerhard 2010. Hintergrundpapier zur Tagung »Politik in der Wachstumsfalle". Loccum.

Schirwitz, Beate 2005. »Wirtschaftswachstum und Beschäftigung - die Beschäftigungsschwelle «, in ifo Dresden berichtet 3/2005, S. 34-37.

Schmidt-Bleek, Friedrich 1997. Wieviel Umwelt braucht der Mensch? Faktor 10, das Maß für ökologisches Wirtschaften. München: dtv.

Schor, Juliet B. 2010. Plenitude. The new economics of true wealth. New York: Penguin Press.

Seidl, Irmi; Zahrnt, Angelika. Hrsg. 2010 a. Postwachstumsgesellschaft. Konzepte für die Zukunft. Marburg: Metropolis.

Seidl, Irmi; Zahrnt, Angelika 2010 b. "Staatsfinanzen und Wirtschaftswachstum «, in Postwachstumsgesellschaft. Konzepte für die Zukunft, hrsg. v. Seidl, Irmi; Zahrnt, Angelika, S. 179-188. Marburg: Metropolis.

Shafik, Nemat; Bandyopadhyay, Sushenjit 1992. Economic growth and environmental quality. Time-series and cross-country evidence. Background paper for World Development Report 1992. Vol. 904, policy research working paper. Washington: The World Bank.

Solow, Robert M. 1968. »Ein Beitrag zur Theorie des wirtschaftlichen Wachstums ", in Wachstum und Entwicklung der Wirtschaft, hrsg. v. König, Heinz, S. 67-97. Köln: Kiepenheuer \& Witsch.

Solow, Robert M. 1974. »The economics of resources or the resources of economics «, in The American Economic Review 64, 2, S. 1-14.

Solow, Robert M. 1997. »Georgescu-Roegen versus Solow/Stiglitz «, in Ecological Economics 22, 3, S. 267-268.

Solow, Robert M.; Wan, Frederic Y. 1974. "Extraction costs in the theory of exhaustible resources ", in The Bell Journal of Economics 7, 2, S. 359-370.

Sommer, Bernd 2011. "Interdependenzen und Ungleichzeitigkeiten im Kontext des anthropogenen Klimawandels ", in Leviathan 39, 1, S. 55-72.

Sorrell, Steven 2007. The rebound effect: an assessment of the evidence for economy-wide energy savings from improved energy efficiency. London: UK Energy Research Centre.

Sorrell, Steven 2010. »Energy, economic growth and environmental sustainability: five propositions ", in Sustainability 2, S. 1784-1809.

SRU (Sachverständigenrat für Umweltfragen) 2011. Wege zur 100\% erneuerbaren Stromversorgung. Sondergutachten. Berlin: Erich Schmidt.

Statistisches Bundesamt 2010. Rohstoffeffizienz: Wirtschaft entlasten, Umwelt schonen. Ergebnisse der Umweltökonomischen Gesamtrechnungen 2010. Wiesbaden: Statistisches Bundesamt.

Stern, Nicholas et al. 2007. The economics of climate change. The Stern review. Cambridge: Cambridge University Press.

Steurer, Reinhard 2004. "Politische Manifestationen einer wissenschaftlichen Auseinandersetzung: Die Wachstumskontroverse im Spiegel der deutschen Wirtschafts- und Umweltpolitik «, in Zeitschrift für Umweltpolitik und Umweltrecht 24, 3, S. 349-390.

Stiglitz, Joseph E. 1974. »Growth with exhaustible natural ressources: efficient and optimal growth paths", in The Review of Economic Studies 41 (Symposium on the Economics of Exhaustible Resources), S. 123-137. 
Stiglitz, Joseph E. 1997. »Georgescu-Roegen versus Solow/Stiglitz «, in Ecological Economics 22, 3, S. 269-270.

Stiglitz, Joseph E.; Sen, Amartya K.; Fitoussi, Jean-Paul 2009. Report by the Commission on the Measurement of Economic Performance and Social Progress. www.stiglitz-sen-fitoussi.fr/en/ index.htm (Zugriff vom 24.04.2012).

SVR (Sachverständigenrat zur Begutachtung der gesamtwirtschaftlichen Entwicklung) 1994. Jahresgutachten 1994/95. Bundestagsdrucksache 13/26.

SVR 2009. Die Zukunft nicht aufs Spiel setzen. Jahresgutachten 2009/2010. Wiesbaden: SVR.

SVR 2011. Herausforderungen des demografischen Wandels. Expertise im Auftrag der Bundesregierung. Wiesbaden: SVR.

SVR; CAE (Conseil d'Analyse Economique) 2010. Wirtschaftsleistung, Lebensqualität und Nachhaltigkeit: Ein umfassendes Indikatorensystem. Expertise im Auftrag des DeutschFranzösischen Ministerrates. Paris, Wiesbaden: SVR, CAE.

TEEB 2010. Die Ökonomie von Ökosystemen und Biodiversität: Die ökonomische Bedeutung der Natur in Entscheidungsprozesse integrieren. Ansatz, Schlussfolgerungen und Empfehlungen von TEEB - eine Synthese (TEEB 2010: The economics of ecosystems and biodiversity: mainstreaming the economics of nature). Malta: Progress Press.

UNEP (United Nations Environment Programme) 2007. Global environment outlook 4: environment for development. Valletta: Progress Press.

UNEP 2010. GREEN economy. Driving a green economy through public finance and fiscal policy reform. Working Paper. Genf: UNEP.

UNEP; IPSRM (International Panel for Sustainable Resource Management) 2010. Assessing the environmental impacts of consumption and production: priority products and materials. Nairobi: UNEP, IPSRM.

Victor, Peter A. 2008. Managing without growth. Slower by design, not disaster. Cheltenham: Edward Elgar.

Victor, Peter A. 2010. »Ecological economics and economic growth ", in Annals of the New York Academy of Sciences 1185, S. 237-245.

von Weizsäcker, Ernst Ulrich; Hargroves, Karlson; Smith, Michael 2010. Faktor Fünf. Die Formel für nachhaltiges Wachstum. München: Droemer.

Wackernagel, Mathis; Rees, William E. 1996. Our ecological footprint: reducing human impact on the earth. Gabriola Island: New Society Publishers.

Walker, Brian; Salt, David 2006. Resilience thinking. Sustaining ecosystems and people in a changing world. Washington: Island Press.

WCED (World Commission on Environment and Development) 1987. Our common future (Brundtland-Report). Oxford: Oxford University Press.

Weimann, Joachim 1994. Umweltökonomik. Eine theorieorientierte Einführung. 3. Aufl. Berlin: Springer.

WWF (World Wide Fund for Nature); Ecofys; OMA (Office for Metropolitan Architecture) 2011. The Energy Report. 100\% Renewable Energy by 2050. Gland: WWF.

WWF; Zoological Institute of London; Global Footprint Network 2010. Living Planet Report 2010. Biodiversität, Biokapazität und Entwicklung. Gland, London, Oakland: WWF, Institute of Zoology, Global Footprint Network.

Zittel, Werner; Schindler, Jörg 2007. Coal: resources and future production. Background paper prepared by the Energy Watch Group. Updated version: 10th July 2007. Vol. 1/07, EWGSeries. Ottobrunn: Energy Watch Group. 
Zusammenfassung: Angesichts der fundamentalen Verunsicherung von Märkten und Menschen durch wirtschaftliche und ökologische Krisen stellt sich die Frage nach den Grenzen des Wachstums mit neuer Dringlichkeit. Dieser Beitrag lässt beide Seiten der aktuellen Wachstumskontroverse zu Wort kommen und setzt sich dabei mit der politischen Debatte ebenso auseinander wie mit ihrer Fundierung in der ökonomischen Theorie. Die Wirtschaftswissenschaften sollten sich den neuen Herausforderungen stellen, etwa der Frage, ob und wie Stabilität und Wohlstand in modernen Marktwirtschaften auch ohne Wachstum erhalten werden können. Auf politischer Ebene kann die Lösung nicht in einer gezielten Beschränkung des Wachstums liegen, sondern muss an den ökologischen Belastungsgrenzen ansetzen. Wir plädieren für eine ökologische Schuldenbremse, welche die Verschuldung in den Umweltsystemen zulasten künftiger Generationen begrenzt.

Stichworte: Wirtschaftswachstum, Umwelt, Nachhaltigkeit, Ökologische Grenzen, Neoklassische Ökonomie, Umwelt- und Ressourcenökonomie, Ökologische Ökonomie

\title{
The natural limits of the economy: a case for an ecological debt brake
}

Summary: With economical and ecological crises unsettling markets and people, the question whether there are limits to growth is back with new urgency. The following article gives voice to both sides of the ongoing growth controversy. It analyses the current political debate, and looks at its foundations in economic theory. We argue that economics should take up the new challenges and explore if and how stability and welfare can be sustained in modern market economies without the necessity of continuous growth. At the political level, actively constraining growth cannot be the solution. Instead, ecological boundaries that must not be transgressed should become the starting point for policy. Therefore, we propose an ecological debt brake that limits the extent to which we borrow from nature and thus impair the chances of future generations.

Keywords: Economic growth, environment, sustainability, ecological limits, neoclassical theory, environmental and resource economics, ecological economics

\author{
Autoren \\ Anna Leipprand \\ Sachverständigenrat für Umweltfragen (SRU) \\ Luisenstraße 46 \\ 10117 Berlin \\ Email: anna.leipprand@umweltrat.de \\ (Corresponding Author) \\ Nils aus dem Moore \\ Rheinisch-Westfälisches Institut für Wirtschaftsforschung (RWI) \\ Büro Berlin \\ Hessische Straße 10 \\ 10115 Berlin \\ Email: nils.ausdemmoore@rwi-essen.de
}

Leviathan, 40. Jg., 2/2012 